\title{
Les diatomées monoraphidées des îles Kerguelen
}

\author{
R. Le Cohu ${ }^{1}$ \\ R. Maillard ${ }^{2}$
}

Aux iles Kerguelen, les monoraphidées sont représentées par les deux genres, Cocconeis et Achnanthes. 35 taxons ont été inventoriés, dont 2 nouveaux pour la science. La plupart des especes et des variétés ont été examinèes au microscope optique, au microscope électronique à balayage et au microscope électronique à transmission : une description détaillée de leur morphologie est souvent donnée. Chez plusieurs especes d'Achnanthes, des vestiges de raphé peuvent être mis en évidence sur la valve sans raphé. Chez dautres especes, toujours sur la valve sans raphé, on observe la présence de pores aux extrémités de l'aire axiale. Ces "pores" peuvent ètre considérés soit comme les vestiges des pores terminaux, soit comme le debut d'un processus vers la formation d'un raphe.

\section{The diatoms * monoraphidinae $*$ in the Kerguelen islands.}

The monoraphidinae, in the Kergue]en Islands, are represented by the two genera, Achnanthes and Cocconeis, producing a total of 35 taxa of which 2 are deemed to be described for the first time. Most of the species and varieties were examined by L.M., S.E.M. and M.E.T.; a detailed account of their morphology is of ten given. Vestigial raphe traces occured in the rapheless valves of several species and varieties. In addition, the presence of "pores" at the ends of the axial area was observed in the rapheless valve of some other species. These "pores" may be considered as vestiges of \&terminal pores " of a former raphe or as precursors of a true raphe that has not yet evolved fully on the rapheless valve.

\section{1. - Introduction}

Jusqu'à maintenant, le travail le plus exhaustif réalisé sur les Diatomées d'eau douce des îles Kerguelen est celui de Manguin (in Bourrelly et Manguin 1954): ce dernier avait inventorié 84 espèces et 62 variétés, dont plusieurs taxons nouveaux pour la science. Le matériel avait été récolté dans 4 stations : bord de la rivière du Port aux Français, lac Simone, lac Marie-Nicole et une tourbière. Antérieurement Germain (1937) avait également étudié quelques échantillons provenant d'une tourbe de phanérogames. Par ailleurs quelques cas particuliers ont été également traités (Germain et Le Cohu 1981, Le Cohu 1982). La présente note porte uniquement sur les Achnanthales; elle entre dans le cadre d'un inventaire des Diatomées d'eau douce des iles Kerguelen, parallèle à celui qui a déjà été effectué pour les aut res algues dulçaquicoles (Thérézien et Couté 1977).

1. Laboratoire D'Hydrobiologie, ERA 702 du C.N.R.S., Univ. P Sábatier. 118 route de Narbonne, F 31062 Toulouse Cedex.

2. 10 bis rec Beaumont, 27000 Evreux.
A part quelques exceptions (mares particulièrement fréquentées par les animaux), les eaux douces des îles Kerguelen peuvent être considérées comme oligotrophes, très pauvres en sulfates, nitrates, phosphates et aussi calcium (Thérézien 1976). Les analyses effectuées pendant une année sur le lac Studer, confirment cette appréciation (Maire, comm. pers.). Comme le signale Tyler (1972), les apports des embruns marins jouent un ròle très important dans la composition minérale des eaux douces de cette région, ceci étant d'autant plus vrai que les plans d'eau sont proches de la mer. Du point de vue des populations diatomiques, il faut remarquer que les espèces purement planctoniques sont relativement rares ; de plus, en eau libre, dans les lacs, tout au moins si l'on se réfère au lac Studer, on n'observe pas de variations saisonnières nettes dans l'évolution annuelle des espèces; il n'y a pas de pic printanier, par exemple.

L'objet de ce travail est l'étude des récoltes faites par Y. Thérézien (de janvier à avril 1973), P. Davaine (en mars 1975) et P. Maire (en 1975 et 1976). Nous donnons ci-apres la liste des localités et leur numéro 
d'ordre en utilisant la même numérotation que celle de Thérézien et Couté (1977): seules sont citées les localités où la présence de Monoraphidées a été observée.

\section{2. - Rivières, ruisseaux, sources, mares, rochers suintants}

\author{
Récoltes effectuées par Y. Thérézien (de janvier à avril \\ 1973): iles Kerguelen - Grande Terre.
}

4TH 31.1 .73 : rivière norvegienne à la limite des secteurs I et Il près de la cabane des hydrobiologistes. Petit bras d'eau stagnante communiquant avec la riviere; berges couvertes d'Acaena.

5TH 31.1.73: zone calme dans le lit de la riviene a proximité du lieu de récolte $n^{\circ} 4$

7TH 31.1 .73 : récolte dans une petite mare très peu profonde (de l'ordre de 10 à $20 \mathrm{~cm}$ ) située à proximité de la cabane des hydrobiologistes prés de la rivière norvégienne.

8TH 1.2.73 : Port Jcanne d'Arc. Petit ravin situé au sud du port. Sur rochers suintants; mousses et algues vertes filamenteuses.

9TH 1.2 .73 ; port Jeanne d'Arc. Récolte dans le même ravin que précédemment, au-dessus des rochers suintants, dans une petite mare du ruisseau presque à sec.

10TH 3.2.73 : petite mare peu profonde à l'ouest de Port aux Français, à proximité d'une cabane abandonnée utilisée pour le marquage des éléphants de mer.

12TH 3.2.73: mare située à l'ouest des deux précédentes. Bordure très riches en algues vertes.

13TH 6.2.73: lit de la rivière du Chàteau, en amont.

14TH 6.2.73 : couverture biologique dans le lit de la rivière du Cháteau.

16TH 6.2.73: rivière du Château. Benthos (périphyton) sur touffes d'Acaena en partie immergées dans une zone de taible courant.

17TH 6.2 .73 : petite mare sur le rebord de la prairie d'Acàna en terrain spongieux à proximité du lit de la rivière du Château.

18TH 6.2.73 : petite mare riche en algues vertes située juste à côté de la précedente.

19TH 6.2.73 : récolte dans le sédiment grossier sur le bord de la rivière. Grattage dans les graviers, avec le filet à planton placé à l'aval.

20TH 6.2.73 : site identique à celui de la récolte précédente. 21 TH 6.2.73 : petit ruisselet tapissé de mousses et d'algues vertes filamenteuses coulant sur un terrain spongieux en aval de la grande cascade de la rivière du Château.

24TH 9.2 .73 : petite mare très abritee, a proximité de la rivière de l'Esi à $4 \mathrm{~km}$ en amont du lac de Marville.

25TH 9.2 .73 : rivière de l'Est a l'ouest du lac de Marville.
26TH 9.2.73: bordure de la rivière de l'Est à l'Ouest du lac Marville.

31TH 11.2.73: rivière des Albatros.

33TH 11.2 .73 : petit ruisseau de la rive gauche de la rivière du Château débouchant dans la rivière près de la cabane qui servait a entreposer le matérie] de pêche électrique.

38TH 24.2.73: rivière du Val de J'Ouest à $400 \mathrm{~m}$ de l'embouchure; courant tres faible.

40TH 24.2.73 : petit ruisseau se jetant dans la baie de Port Elizabeth en face du Mont Bayley : récolte d'algues sur les cailloux.

42TH 24.2.73 : récoltes d'algures poussant sur les pierres dans le courant de la riviere du charbon à 300 m environ en amont de l'embouchure.

46TH 4.3.73: source chaude "Lily", rive nord du lac Tristan.

49TH 7.3.73 : petite mare se trouvant à proximité du ruisseau qui arrive dans l'étang de la charge.

56TH 13.3 .73 ; cuvettes dans un petit ruisseau situé près de Port Douzième, sur la presqu'ile Ronarc'h

57TH 17.3.73: rochers suintants situés près du Port Douzieme

58TH 21.3 .73 : petite mare temporaire à fond enherbé située tout près du Biomar (laboratoire de biologie marine situé sur la base en bordure de mer).

59TH 22.3 .73 : petite mare assez profonde dans le lit de la riviere du Sud.

$60 \mathrm{TH} 22.3 .73$ : courant de la rivic̀re du Sud en aval de la cabane du limnigraphe.

62 TH 24.3 .73 : rochers suintants sur les pentes du mont Crozier vers $700 \mathrm{~m}$ d'altitude.

$69 \mathrm{TH} 27.3 .73$ : source donnant naissance au ruisseau allant se jeter dans le lac Margot.

71TH 27.3.73: vallée supérieure de la rivière du Sud à proximité du col des tourbières. Récolte de Nostoc en thalles bruns sur le sol gorgé d'eau.

72TH 28.3.73: ruisseau du Borgne.

73TH 7.4.73 : petil muisseau aboutissant dans la baie de Lar mor. Péninsule Rallier du Baty.

74TH 7.4 .73 : région du glacier Ampere : petite mare creusee dans le roc, alimentée par le ruissellement (ne provenant pas du glacier) et située à environ $400 \mathrm{~m}$ du front du glacier Ampere.

- Récoltes d'algues effectuées par P. Davaine (en mars 1974) : iles Kerguelen - Grande Terre.

2DA 20.3.74 : ruisseau affluent rive droite du Val Sombre. Presqu'ile Jeanne d'Arc. Récoltes sur rochers suintants.

3DA 16.3 .74 : rivières des Maraconis, Presqu'île Ronar'h Récolte sur blocs et gajets du fond, juste en amont de l'embouchure.

4DA 23.3 .74 : rochers suintants en altitude sur les falaises ouest du lac de Guilvinec - plateau central.

5DA 19.3.74 : ruisseau de Mont Fergusson dans les falai ses sur la rive gauche du Val du Levant. Presqu'ile Jeanne d'Arc. Récolte sur galets. 
6DA 17.3.74 ; ruisseau à l'ouest de l'abri Saint-François. Presqu'ile Ronarc'h. Récolte sur les galets du fonds. Altitude $200 \mathrm{~m}$ environ.

7DA 19.3.74 : rivière Alberic dans le canyon des sourcils noirs. Presqu'île Jeanne d'Arc. Récolte sur blocs au confluent avec le principal affluent rive droite.

8DA 23.3.74 : gave de l'Azorella en amont du lac Guilvinec - plateau central. Récolte sur les galets du fond.

9DA 23.3.74 : rivière Valdotajne en haut de la cascade Bossière - plateau central. Récolte sur galets.

10DA 19.9 .74 : ruisseau dans les falaises de la baie de l'A Pic. Presqu'île Jeannne d'Arc. Récolte sur cailloux en altitude.

\section{- Récoltes effectuées par $P$. Maire.}

[4MA \& 109MA 27.4/7.12.75: rivière des Américains ; plancton et benthos.

23MA, 24MA, 51MA, 94MA \& 123MA 23.5/23.6/31.7/7.11/5.1.75: rivière du Château; plancton et benthos.

64MA 6.9.75: rivière du Dôme rouge

22MA, 52MA, 53MA, 79MA, 80MA, 11MA \& !12MA $28.5 / 1.8 / 1.8 / 9.10 / 9.10 / 15.12 .75$ : rivière de la Ferme ; plancton et benthos.

96MA 21.11.75: rivière des galets

5MA, 13MA, 31 MA, 32MA, 55MA, 56MA, 131MA \& 132ma 15.2/24.2/20.4/18.6/3.8/3.8/14.12/14.12.75: riviere du Sud ; plancton et benthos.

\section{3. - Lacs}

\section{- Récoltes effectuées par P. Maire.}

135MA 8.2.75: lac Alicia: plancton et benthos.

138MA 7.7/2.10/5.12.75: lac des Stemes; plancton et benthos.

$139 \mathrm{M} \mathrm{A}$

9.3./23.3/19.4/20.5/16.6/26.7/21.8/18.9/7.10/30.10/2.12/75: lac

Studer; plancton et benthos.

140MA 2.4/9.5/14.6/24.7/13.8/27.9/23.10/29.11.75;6.1.76: lac des Korrigans; plancton et benthos.

Pour chaque taxon, le numéro des stations est indiqué de la façon suivante: $74 \mathrm{TH}$ (récolte Thérézien); 2DA (récolte Davaine); 14MA (récolte Maire).

Les notations (T,C.) : très commun ; (C.) : commun ; (A.C.) : assez commun ; (A.R.) : assez rare ; (R.) : rare; (T.R.) : très rare, indiquent la notion de fréquence dans les récoltes.

Autres abréviations utilisées : M.E.B., microscope électronique à balayage : M.E.T., microscope électronique à transmission.

\section{4. - Liste des espèces. Observations et discussion 1}

Aux îles Kerguelen, les Monoraphidées sont représentées par les genres Cocconeis et Achnanthes. 35

1. Les récoltes en provenance des lacs ont été étudiées par $\mathbf{R}$. Le Cohu, celles de tous les autres milieux par R. Maillard. Les observations au microscope électronique ont été réalisées par $\mathbf{R}$ Le Cohu taxons, dont 2 nouveaux pour la science, ont été identifiés. Cette liste est sans doute loin d'être exhaustive, plusieurs espèces trés petites nécessitant une étude plus approfondie.

Cocconeis Ehrenberg.

- Cocconeis feuerbormii Hust.

Espèce décrite par Hustedt (1937-1938) aux îles de la Sonde, retrouvée par Carter et Denny (1982) en Sierra Leone ; par Coste et Ricard (1982) dans l'île Maurice et aux Seychelles. Pour le moment, sa disIribution semble limitée à l'hémisphère sud.

4TH (présent) ; 139MA, 140MA (R.).

- Cocconeis pediculus Ehr.

IOTH (présent) ; 139MA, 140MA (R.).

- Cocconeis placentula Ehr.

10TH (présent) ; 135MA, 139MA, 140MA (T.R.).

- Cocconeis scutellum Ehr.

Forme marine, présence sans doute accidentelle. 10TH (T.R.) ; 138MA, 140MA (T.R.).

. C. scutellum var, ampliata Grun. 10TH (T.R.).

- Cocconeis therezieni nov. sp. (Fig. 1, 2, 29, 30 à 37, 56, 84 à $97,189,190$ ).

Elliptica valva, apicibus late rolundatis; lata 7-11 ${ }_{\mu} m$, rarissime $30{ }_{\mu} m$ - Raphovalva : raphe summà linea, recta; axiale angustissima a red, centrale areà cum transversa fascia quae valvae latitudinis dimidium facit; omnibus obliciis radiantibus striis, medianes 20-24 in $10{ }_{\mu} \mathrm{m}$, ceterae 29-30 in $10 \mu \mathrm{m}$, deinde magis magisque densis usque ad extremitates, omnibus leniter punctatis praeter eas quae circumdant centralem aream enormiter granditer punclatas. - Areovalva : area axiale angustissima, aliquander leniter lanceolata, nullo centrale area. Strits obliciis magis magisque curvis ad apices, 19-21 in $10 \mu m$ usque ad polos, maxime aequalite rque punctatis, $12-16$ in $10 \mu \mathrm{m}$. Vicissima Cocconeis feuerbornii Hust., cum eà confundi potest est sed maxime dis. cemitur constructionae valvae cum raphe quae in ea multum lenis est.

Valve elliptique, extrémités largement arrondies : longueur : $12-19 \mu \mathrm{m}$, exceptionnellement $30 \mu \mathrm{m}$, largeur: $7-11 \mu \mathrm{m}$.- Valve à raphé : raphé filiforme, droit, aire axiale très étroite, aire centrale en bande transversale, la moitié de la largeur de la valve. Stries transapicales toutes radiantes, les médianes 20-24 en $10 \mu \mathrm{m}$, les autres 28-30 en $10 \mu \mathrm{m}$, de plus en plus serrées jusqu'aux extrémités, toutes délicatement ponctuées, sauf celles qui entourent l'area 
centrale, souvent irrégulièrement plus grossièrement ponctuées. - Valve sans raphe : aire axiale très étroite, quelquefois légèrement lancéolée : aire centrale nulle. Stries transapicales de plus en plus courbées vers les poles, 19-21 en $10 \mu \mathrm{m}$ jusqu'aux poles, fortement et régulièrement ponctuèes, 12-16 points en $10 \mu \mathrm{m}$.

Espèce très proche de Cocconeis feuerbormii Hust. avec laquelle elle peut être confondue ; elle s'en distingue surtout par la structure beaucoup plus délicate de la valve à raphé mais aussi par la forme de l'aire centrale. La valve sans raphé des plus grands individus (vue interne, fig. 37) présente également des similitudes avec Cocconeis placentula var. euglypta (Ehr.) cleve.

\section{Structure au microscope électronique}

- Valve à raphé : au M.E.B., en vue externe (fig. 84 à 88 ), les stries se résolvent en une seule rangée de ponctuations (aréoles, 25-30 en $10 \mu \mathrm{m}$ ) ouvertes vers l'extérieur; souvent, ce qui est déjà visible au microscope photonique, au niveau de l'aire centrale, les ponctuations s'allongent, deviennent subquadrangulaires (M.E.T., fig. 189). Les stries s'interrompent au niveau de la marge valvaire saillante ; elles reprennent sur le manteau sous la forme de simples ponctuations, seulement visibles sur les valves débarassées des ceintures connectives (fig, 84/85, 89). En vue interne, les ponctuations, plus grossières qu'en vue externe, apparaissent fermées (présence d'un velum difficilement mis en évidence au M.E.T.) et sises au fond de petites dépressions (fig. 91). Les deux branches du raphé se trouvent au fond d'une gouttière fortement silicifiée : elles s'infléchissent au sens opposé au niveau de l'aire centrale.

- Valve sans raphé : en vue externe (Fig. 92 à 96), les stries, comme sur la valve à raphé, sont constituées d'aréoles ouvertes vers l'extérieur. L'aire axiale s'élargit légèrement dans sa partie médiane (fig. 95/96). Les stries, interrompues sur les bords marginaux de la face valvaire, reprennent sur le manteau sous la forme d'une seule ponctuation (fig 92,96). En vue interne comme sur la valve à raphé, les ponctuations sont plus grossières qu'en vue externe. L'aire axiale se marque par une arête fortement silicifiée faisant saillie à l'intérieur de la valve (fig. 97).
4TH, 7TH, 14TH, 20TH (T.R.) ; 5DA, 38TH, 23MA, 49MA, 94MA, 111MA (présent) ; 139MA (C.); 138MA (A.R.) ; 140MA (A.R.).

\section{Achnanthes Bory.}

- Achnanthes abundans Manguin (in Bourrelly et Manguin 1954). (fig. 38 à 41,98 à 108, 191).

Dans les quatre lacs étudiés, cette espèce est très fréquente et parfois méme abondante. Elle se trouve souvent associée avec des algues vertes filamenteuses et des Cyanophycées. C'est une forme plutôt lit. torale mais que l'on peut également trouver en eau libre. Dans les autres milieux prospectés, elle a été rencontrée dans une seule station.

7TH (C.) : 138/135MA (C.) : 139/140MA (T.C.).

Au M.E.B., comme le signale d'ailleurs Manguin (1954) dans sa diagnose, l'aire centrale de la valve à raphé apparaît irrégulière comme le montrent les figures 98/99, 101, 104/105, 107. Les stries sont cons. tituées d'une seule rangée de ponctuations (aréoles ?). En vue interne, au niveau de l'aire centrale, les deux branches du raphé se terminent par un très court crochet dirigé vers le bord valvaire (fig. 99). La valve sans raphé présente la même irrégularité de l'aire centrale que la valve à raphé. Les stries se résolvent également en une seule rangée de ponc. tuations. En vue interne, celles-ci sont entourées d'un épaississement fortement silicifié (Fig. 100. 102). Sur un cerain nombre d'individus, il faut aussi noter la presence d'un pore en forme de fente allongée, au niveau où serait placé l'hélictoglosse (fig. 108). Manguin (1954) avait aussi décrit une var. ellip. tica de Achnanthes abundans, Les nombreuses inves. tigations effectuées au M.E.B., au sein d'une même population. montrent que toutes les formes de passage existent entre l'espèce et la variété. Cette dernière représente la limite "inférieure" de l'espèce : la diminution de la taille entraine une altération de la forme, notamment du caractère "tumide" de la partie médiane de la valve, et un resserrement de l'aire centrale, parfois à peine marquée (fig. 105). Ces caractères sont évidemment souvent très difficiles à distinguer au microscope photonique. Pour toutes ces raisons, la var. elliptica ne semble pas devoir être conservée. La diagnose de Manguin (1954) doit donc être modifiée: "valves linéaires, légèrement tumides dans la partie médiane devenant elliptique chez les petites formes. Longueur : 6,5-18 $\mu \mathrm{m}$; lar- 
geur : $3,5.5 \mu \mathrm{m}$. Valve à raphé avec une aire centrale irrégulière, le plus souvent circonscrite par des stries très courtes ( 2 au maximum) qui peuvent sembler absentes au microscope photonique. Valve sans raphé avec une aire axiale plus ou moins lancéolée s'élargissant en une aire centrale irrégulière. Stries transapicales radiales, $28-32$ en $10 \mu \mathrm{m}$ (le plus souvent 30$)$ ".

Achnanthes adundans est proche de Achnanthes reimeri Camburn et Al. (1978) dont Lange-Bertalot et Ruppel (1980) ont fait un synonyme de A. minutissima. Les figures 315 et 316 de cette espèce, données par les deux auteurs précités montrent également de grandes similitudes avec A. abundans (fig. 191). Cette espèce se rapproche également beaucoup des figures d'A. minutissima (fig. 35 à 40) données par Schoeman et Ashton (1982) mais elle s'en distingue nettement par la forme de l'aire centrale.

- Achnanthes austriaca Hust. (fig. 42/43, 109 à 112). Espèce rare dans les lacs, excepté celui des Korrigans. Elle n'a pas été observée dans les autres milieux prospectés.

135/138/139MA (R.) ; 140MA (A.C.).

Au M.E.B., seules des valves sans raphé ont pu être observées. En vue externe (fig. 111/112), les stries se résolvent en une seule rangée de ponctuations plus ou moins subquadrangulaires $\# 40$ en $\left.10{ }_{\mu} \mathrm{m}\right)$; celles-ci, interrompues au niveau de la marge valvaire, reprennent sur la manteau sous la forme de fentes allongées. Aux extrémités de l'aire axiale, on note la présence d'un pore (fig. 109, 111, 112).

. Achnanthes austriaca var. helvetica Hust. (fig. $113,114)$.

Les stries de cette variété semblent plus finement ponctuées que celles de l'espèce type, ce qui semble confirmé par les observations de Leclercq et Fabbri (1982).

- Achnanthes coarctata (Bréb.) Grun. (Fig. 57).

Oligohalobe, aérophile. Cosmopolite.

7TH (R.) ; 9TH (présent) ; 139MA (R.).

. Achnanthes coarctata var, elliptica Krasske (fig. 3 à $5,61,115$ à 118). Dimensions : 30-50 × 10-12 ${ }_{\mu} \mathrm{m}$; nombre de stries : $11-14$ en $10{ }_{\mu} \mathrm{m}$. 139MA (A.R.).

Dar rapport à la variété type (Hustedt 1959, fig. $872 \mathrm{~d} / \mathrm{e}$ ), les valves sont plus lancéolées, les extré. mités nettement moins obtuses.
Au M.E.B., en vue externe, sur l'hypovalve (fig. 115), les deux branches du raphé présentent des fissures terminales et des pores centraux incurvés dans la même direction. Les stries sont constituées d'une seule rangée de grosses ponctuations (sans doute des aréoles). En vue interne (fig. 116), les deux branches du raphe se terminent, au niveau du nodule central, par des boucles non fermées comme Achnanthes inflata (fig. 130). Au niveau de l'aire centrale (vues externe et interne), on observe la présence d'un stauros at teignant toujours les bords valvaires, contrairement à ce qui sera observé pour Achnanthes inflata.

Sur l'épivalve, le pseudo-raphé se trouve complètement déjeté sur le côté (fig. 117/118). Les stries, constituées de grosses ponctuations séparées par des cótes fortement silicifièes, se prolongent sans interruption sur le manteau de la valve, type de structure que l'on retrouve chez $A$. inflata. En vue interne, aux deux extrémités de la valve, on note la présence de ce que $\mathbf{M c}$ Intire et Reimer (1974) ont appelé un uorbiculus»; il se place à la terminaison du pseudoraphé.

- Achnanthes confusa Manguin (in Bourrelly et Manguin 1954). (fig. 18 à 21, 44, 119 à 121, 124, 192 à 195).

Espece présente seulement dans les lacs, souvent fréquente. Elle se localise dans le même type d'habitat que Achnanthes abundans.

135/138MA (A.C.) ; 139/140MA (C.).

Quelques additifs doivent être apportés à la diagnose de Manguin (1954) : longueur : $10-18 \mu \mathrm{m}$ au lieu de $15-16 \mu \mathrm{m}$; nombre de stries, jusqu'à 34 en $10 \mu \mathrm{m}$ au lieu de 28.30. Au microscope photonique, les stries de la valve sans raphé apparaissent nettement ponctuees.

Au M.E.B. et au M.E.T., les caractères les plus distinctifs sont fournis par la valve sans raphé. Les stries ont une structure très particulière. Chacune d'entre elle débute par une aréole marginale bacilliforme ; elle est suivie de deux autres (rarement trois) beaucoup plus courtes (fig. 120.124, 193 a 195). Les stries s'interrompent sur les bords de la face valvaire puis reprennent sur le manteau (fig. 124). Ce qui esı déjà visible au microscope photonique, l'aire centrale se marque par une ou deux stries nettement raccourcies, réduites à l'aréole 
marginale. Tous les spécimens observés présentent un pore au niveau oủ serait placé le nodule terminal (Fig. 121, 194).

. Achnanthes confusa var. atomoides Manguin (fig. 45/46, 122/123, 125, 196/197).

Variété rencontrée uniquement dans les lacs, comme l'espèce type ; elle a le même type d'habitat que cette dernière.

135/138MA (A.C.) ; 139/140MA (C.).

En ce qui concerne les dimensions, la diagnose de Manguin (1954) doit être modifiée. Longueur : 7,5-16 au lieu de $11,25 \mu \mathrm{m}$; largeur : $3-5 \mu \mathrm{m}$ au lieu de $3.75 \mu \mathrm{m}$; nombre de stries ; $26-30$ en 10 ${ }_{\mu} \mathrm{m}$. De plus, il faut ajouter que sur les valves sans raphé, les stries apparaissent nettement ponctuées (fig. 45/46).

Au M.E.B. et au M.E.T. (fig. 122/123, 125 , 196/197), les valves sans raphé ont le même type de structure de stries que l'espece type. Deux critères permettent à coup sûr de distinguer l'espèce de la variété : l'un déjá visible au microscope photonique, aire centrale non élargie dans la partie médiane: l'autre, par contre, seulement mis en évidence au M.E.B. et au M.E.T., absence de pore au niveau où serait placé le nodule terminal.

- Achnanthes conspicua A. Mayer var. brevistriata Hust. (fig. 6/7).

\section{5/138/139/140MA (R.).}

. Achnanthes delicatula (Kütz.) Grun. ssp. delicatula in Lange-Bertalot et Ruppel (1980). (fig. 10/11, 47/48).

138MA (R.); 139/140MA (T.R.).

Ce n'est pas sans hésitation que les types représentés par les figures citées ont été attribués à A. delicalula, margré quelques petites différences du point de vue morphologique ; de plus, la valve à raphé présente de grandes similitudes avec $A$. holstii Cleve. Cette espèce étant plutôt rare dans les milieux prospectés, il n'a pas été possible de l'examiner au microscope électronique. Ce nom est donc avancé sous toute réserve.

- Achnanthes delicatula ssp. hauckiana (Grun.) L.B. et Ruppel (1980). (fig. 12/13, 14 ?, 15 ?, 16 ?. $17 ?, 126,218$ à 221).

138MA (R.) ; 139/140MA (T.R.).
Les figures $12 / 13$, peuvent être rapportées à cette sous-espèce telle que l'ont définie Lange-Bertalot et Ruppel (1980). Les plus petites formes (fig. 14 à 17) peuvent prêter à confusion ; la longueur peut descendre jusqu'à $5,5 \mu \mathrm{m}$ pour une largeur de 3 $\mu \mathrm{m}$; le nombre de stries est nettement plus élevé que pour la ssp hauckiana. Elles pourraient correspondre à la rigueur à $A$. delicatula tel que le représente Germain (1981) avec les figures $14 \mathrm{et}$ 15 (pl. 42). Cependant, au M.E.B., ces petites formes montrent une structure des stries différentes. Les figures $126(8 \times 3,4 \mu \mathrm{m}, 16$ stries en 10 $\mu \mathrm{m}), 219 / 220$ montrent une structure des stries avec 5 rangées d'aréoles correspondant avec celle donnée par Lange-Bertalot et Ruppel (1980) pour la ssp. hauckiana; toutefois, le nombre de stries, rappelons-le, est beaucoup plus élevé que pour cette dernière (7.5-10 en $10 \mu \mathrm{m})$. Un seul exemplaire ayant pu être observé au M.E.B., il est difficile de modifier la diagnose des deux auteurs précités. Les figures $127(6,5 \times 3,5 \mu \mathrm{m}, 18$ stries en $10 \mu$ ) et 223 présentent de grandes analogies avec $A$. engelbrechtii Cholnoky tel que le figurent Schoeman et Ashton (1983, fig. 3). Pour les petites formes, de longueur inférieure à $10_{\mu} \mathrm{m}$, il est donc impossible de distinguer au microscope photonique celles qui appartiendraient soit à la ssp. hauckiana, soit à $A$. engelbrechtii.

. Achnanthes delicatula var. australis Manguin (in Bourrelly et Manguin 1954).

123MA (R.); 10/25TH (T.R.); 3DA, 72TH (présent).

Il faut remarquer que les figures 11 et 47 de la valve sans raphé d'A. delicatula trouvé dans les lacs correspondent parfaitement bien avec celle de la variêté de Manguin (1954).

- Achnanthes engelbrechtii Cholnoky (fig. 14?, 15 ?, 16 ?, 17 ?, 127, 223).

138MA (R.).

Pour cette espèce, se reporter à a discussion concernant la ssp. hauckiana.

- Achnanthes flexella (Kütz.) Brun.

135MA (T.R.).

- Achnanthes germainii Manguin (in Bourrelly et Manguin 1954). (fig. 8/9.) 6MA, 25TH (R.); 14/16/20MA, 5/23/56/131/132MA (T.R.); 5/19TH (présent) ; 139/140MA (T.R.).

Longueur : $16-19 \mu \mathrm{m}$; largeur : 6-8,5 $\mu \mathrm{m}$. 
Dans les lacs, l'espèce type a été rencontrée ; dans les autres milieux prospectés. généralement 4 a 6 stries unilatérales sont présentes au niveau de l'aire centrale de la valve à raphé, au lieu de 2 très écartées comme l'indique Manguin (1954).

- Achnanthes gibberula Grun. (fig. 25/76) in LangeBertalot et Ruppel (1980). 138/139/140MA (R.).

- Achnanthes inflata (Kütz.) Grun. (fig. 22/23, 59 à 61,128 à 135).

Espèce oligohalobe, aérophile, rhéophile Cosmopolite.

62 TH(T.C.dominant);2/7DA(R.) : 4/13/14/16/19/25/33/42/69/72/74TH, 5/10DA, 6/94MA (T.R.)： 5/9/38/57TH, 3DA, 49/56MA (présent); 139MA (A.C.) ; 138/140MA (T.R.).

Dimensions : $30-80 \times 16-23 \mu \mathrm{m} ; 9.11$ stries en 10 $\mu \mathrm{m}$.

L'espèce type, avec trois fortes dilatations, n'a jamais été rencontrée. Le plus souvent, deux varia. tions ont été observées, l'une plus ou moins rhombique, l'autre plus ou moins citriforme. Ces deux variations se rapprochent beaucoup de la variété elata (Leud. - Fortm.) Hust. telle que la figure Germain (1981) et pourraient sans doute y être rapportées. Si la largeur est à peu près constante sur tous les spécimens observés, la longueur est très variable ; parfois, le rapport Longueur/largeur ne dépasse pas 2. Très souvent le stauros n'atteint pas les bords de la face valvaire ( $f i g .22 / 23,128$ ).

Au M.E.B., aucune vue externe de la valve à raphé n'a pu être observée. En vue interne (fig. 128), les stries, constituées d'une seule rangée d'aréoles, sont séparées par des côtes fortement silicifiées. Chaque aréole présente un crible avec des pores disposés en rangées parallèles (Fig. 130). Caractère déjà nettement visible au microscope photonique, l'aire centrale est souvent limitée par 3 ou 4 stries nettement écourtées. Au niveau du nodule central, les deux branches du raphé se terminent par une boucle presque fermée (Fig. 130), comme celle de A. coarctata var. elliptica.

Sur la valve sans raphé, le pseudo-raphé est complètement déjeté sur le côté (fig. 133/134); par suite, surtout en vue externe (fig. 131/132) et suivant la position du frustule, elle peut se révéler très difficile à distinguer. Les stries, en vue externe, sont constituées d'une seule rangée de grosses aréoles subcirculaires (fig. 131/132). L'arête marginale est irrégulièrement dentelée (fig. 132). A chaque pôle de la valve, suivant la terminologie adoptée par Mc Intire et Reimer (1974), on note, comme chez A. coarctata var. elliptica, la présence d'un "orbiculus». Celui-ci est fermé par une mince membrane (fig. 131/132) qui peut disparaitre lors de la préparation à l'acide; lorsqu'elle subsiste, la présence de pores n'a jamais pu être mis en évidence. A chaque pôle, "l'orbiculus" est plus ou moins connecté avec le pseudo-raphé (fig. 134/135), comme l'avait déjà signalé Mc Intire et Reimer (1974) pour d'autres Achnanthes du même groupe. A l'intérieur de la valve, les stries sont séparées par de grosses côtes fortement silicifiées ; de chaque côté du pseudoraphé, stries et côtes se prolongent sans interruption sur le manteau (fig. 133/134); ces dernières se terminent par des indentations sur lesquelles s'insère la plaque de suture (fig. 135). Les éléments de la ceinture connective (observés sur un seul spécimen) montrent chacun, en vue interne, une double rangée de pores (fig. 129).

- Achnanthes lanceolata (Bréb.) Grun. (fig51, 136). 5/38TH,5DA(C) ;2DA, 13/20/57/62TH (A.C.) ; 4/56TH (A.R.); 5/10DA. 51/56/123MA, 59/62/72TH (R.) ;6DA, 36/37/49/50/93/94/122/131/132MA, 9/10/14/18/31/33/42/46/49/59TH (T.R.) ; 8TH (présent) ; 135/138/139/140MA (A.R.).

Achnanthes lanceolata var. lanceolatoides (Sov.) Reim. (fig, 52 à 55, 137 à 146, 198 à 200).

Décrit aux Etats-Unis par Sovereign (1958) sous le nom d'Achnanthes lanceolatoides. ce taxon a été retrouvé par Pierre (1977) dans l'île de la Possession (Archipel des Crozet); Coste (1978) donne également une vue externe de la valve sans raphè d'Achnanthes lanceolata (pl. 7, fig. 1) qui pourrait s'y rapporter. Aux îles Kerguelen, ce taxon est pratiquement rencontré dans tous les habitats, avec naturellement des varia. tions dans sa fréquence.

5DA, 21 TH (T.C.) ; 139/140MA (C.); 138MA (A.R.) ; 135MA (R.).

Que ce soit au microscope photonique ou électronique, la structure des stries, de part et d'autre de l'aire centrale, constitue le caractère le plus typique de cette variété. Cependant, par rapport à la description de Patrick et Reimer (1966), des variations ont été notées.

Sur la valve à raphé, les stries, délimitant l'aire centrale, présentent une grande variabilité et 
une grande irrégularité de structure ; cela peut même être observé sur une même valve (fig. 137 à 140,198 ). Sur la valve sans raphé, l'irrégularité de la structure des stries se retrouve au niveau de celles qui entourent le renflement médian : les stries peuvent être "normales " ou telles que le montrent les figures 142 a 145, 199 et 200. L'origine des stries "anormales" doit sans doute être un processus de silification intense entrainant le comblement de tout ou partie des stries. Par ailleurs, du point de vue structurel, rien ne distingue la variété de l'espèce.

Au M.E.B. et M.E.T., les stries se résolvent le plus souvent en trois lignes de pores, parfois quatre sur la valve à raphé (fig. 141, 198). En vue externe, sur la valve sans raphé, l'aire axiale constitue un sillon net tement marqué (fig. 143) ; la face valvaire est délimitée par une série d'indentations (fig. 143) plus ou moins interrompues au niveau des stries, lesquelles semblent se prolonger sur le manteau. Le renflement médian peut être défini comme un sinus (fig. 146), type de structure qui serait, selon Moss et Carter (1982), une des caractéristiques d'Achnanthes lanceolata. Il faut d'ailleurs signaler que, dans ce cas, les figures (Taf. 946) données par Gerloff et Helmincke (1977) ne représentent pas Achnanthes lanceolata mais A. rostrata Ostrup.

- Achnanthes lapponica Hust. var. ninkei (Guerm. et Mang.) Reim.

(Syn.: Achnanthes aretasii Manguin). (fig. 24, 62 à 64,147 à 149.)

La diagnose donnée par Patrick et Reimer (1966) a été adoptée en apportant de légères modifications en cc qui concerne les dimensions : longueur, 9-27 $\mu \mathrm{m}$ au lieu de 9-24; largeur, 4-8,5 $\mu \mathrm{m}$ au lieu de 5-7. L'espèce Achnanthes aretasii (fig. 24, 149) de Manguin (1954) représente, selon nous, les grands spécimens de la variété ninkei. Chez ceuxci, la valve est plus élancée, les extrémités plus étirées, les stries au niveau de l'area centrale plus écourtées que sur le type originel (fig. 10/11, Guermeur et Manguin, 1953). Il faut signaler que les deux types se retrouvent au sein d'une même population. Le type $A$. aretasii (fig. 24, 64, 149) montre beaucoup d'analogies avec $A$. investians; cette espèce décrite par Carter (1966), dans l'île Tristan da Cunha, est légèrement plus petite $(7-10 \times 3-6$ $\left.{ }_{\mu} \mathrm{m}\right)$ et a un nombre de stries légèrement supérieur (28-34) en $10 \mu \mathrm{m}$. On peut toutefois penser que c'est un seul et même taxon.

10DA (A.C.) ; 31TH (R.) ; 8DA, 4/13/14/16TH, 24MA (T.R.) ; 19/58TH (présent) ; 140MA (A.R.); 135/138/139MA (T.R.).

Au M.E.B., seules des vues internes de la valve à raphé (fig. 147) ont pu être observées. Les stries sont très finement ponctuées. L'aire centrale, hémicirculaire est bordée d'un côté de stries raccourcies en nombre variable ( 5 sur le type originel ninkei, 10 sur le type aretasii); elle est largement ouverte jusqu'au bord de la valve, du cóté opposé. La valve sans raphé ( $f$ ig. 148/149) présente le même type d'aire centrale que la valve à raphé. Sur tous les spécimens observés, on note la pré. sence d'une gouttière le long de l'aréa axiale, gouttière qui stoppe au niveau de l'aire centrale.

- Achnanthes manguinii Hust. (fig. 65, 150 à 153).

Espèce décrite par Hustedt (1952), Iles Kerguelen.

25/26TH (A.C.) ; 20TH (A.R.) ; 4/7DA, 31/33/56 TH, 23/56MA (R.) : 2/3/5 10DA, 9/14/17/19/42/49/60,62TH, $5 / 6 / 14 / 24 / 36 / 37 / 49 / 50 / 80 / 93 / 112 / 122 / 131 / 132 \mathrm{MA}$ (T.R.) ; 7/8/16/18/57TH, 96/123MA (présent) ; 135MA (T.R.) : 138/139/140MA (R.).

Au M.E.B., seules des valves à raphé en vue interne ont pu être observées (fig. 150). Au niveau de l'aire centrale, les spécimens rencontrés montrent toujours 6 a 8 stries nettement raccourcies, stries attribuées à la valve sans raphé par Manguin (1954). Les stries sont constituées d'une seule rangée de ponctuations. Au niveau du nodule central, les deux branches du raphé se terminent par des crochets dirigés en sens opposé, vers la marge valvaire. Sur la valve sans raphé, l'aire axiale est telle que décrite par Manguin (1954), c'est à dire lancéolée (fig. 151/152). Les stries sont constituées d'une seule rangée de ponctuations. Chez un certain nombre d'individus, un pore a pu être mis en évidence à l'extrémité de l'aire axiale (fig. 151,153).

. Achnanthes manguinii var. elliptica Manguin (in Bourrelly et Manguin 1954). (fig. 66,154).

Plus rare que l'espece dans tous les biotopes où elle a été rencontrée.

4DA (R.) ; 139/140MA (T.R.).

Les valves ont des bords parallèles ou convexes : par ailleurs, il n'y a pas de différence avec l'espèce. 
- Achnanthes marginulala Grun. (fig. 67 à 69, 155). Espèce assez rarement rencontrée, uniquement dans les lacs.

\section{MA (A.R.) ; 135/138/139MA (T.R.).}

Quelques valves sans raphé ont pu être observées au M.E.B. : les strjes se résolvent en une seule rangée de ponctuations (fig. 155). Par rapport à la figure publiée par Leclercq et Fabbri (1982), la seule différence réside dans l'absence d'un sillon au niveau de l'aire axiale.

- Achnanthes minutissima Kütz. (fig. 156 à 161 . 201 à 205).

6DA, 57/74 TH, 22/64MA(T.C.);3/8/9/10DA, 14/20/25/26TH, 22/23/24/36/111/112MA (C.) ; 5/8DA $9 / 16 / 19 / 31 / 40 / 42 / 72 \mathrm{TH}$. 13/14/31/32/51/79/80/112/123MA (A.C.) ; 38/42/56TH (A.R.) ; 3/4/5DA ; 4/33/59/60TH, 6/56/109/131/132MA (R.) ; 2/10DA, 8/9/18/33/49/59 TH, 37/49/50/79/80/93/121/122/123MA(T.R.) ; 139/140MA (A.C.) ; 135/138MA (A.R.)

Les propositions de Lange-Bertalot et Ruppel (1980), regroupant sous le nom d'Achnanthes minutissima, notamment $A$. affinis Grun., A. linearis (W. Sm) Grun, et A. microcephala (Kùtz.) Grun., ont été adoptées. Naturellement, cette espèce présente une grande variabilité avec différentes formes de passages.

Différents types ont été rencontrés, souvent au sein d'une même population :

. spécimens (fig. 156, 201/202) dont la valve à raphé présente une interruption unilatérale des stries formant une aire centrale asymétrique : ils correspondraient à $A$. microcephala tel que l'a défini Germain (1981).

- spécimens morphologiquement identiques ou très proches des précédents, avec une aréa centrale symétrique ( $f$ ig. 157 ) ou avec un stauros (fig. 158)

- spécimens à valves linéaires atténuées et arrondies aux extrémités, avec un stauros (fig. 159). La figure 160 pose un problème ; elle représente sans doute une valve à raphé d'A. confusa Mang. mais pourrait être également assimilée à $A$. minutissima

. spécimens à valves elliptiques (rhombiques) arrondies-obtuses aux extrémités (fig. 161, 205).

- Achnanthes modesta Manguin (in Bourrelly et Manguin 1954). (fig. 70 à 74, 162 à 165, 206 à 208).
135MA (R.): 138MA (A.R.) ; 139/140MA (C.). Manguin (1954) dans la diagnose de cette espèce, décrit la valve à raphé comme "ayant une aire axiale étroite, à peine élargie au centre en une aire centrale irrégulière, très peu étendue, parfois absentew. Selon nos observations, l'aire axiale de la valve à raphé présente souvent, des deux côtés, une strie légèrement raccourcie et plus écartée des autres (fig. 73, 163), parfois une seule strie d'un côté, aucune de l'autre (fig. 72), parfois un stauros très étroit (fig. 74). Sur la valve sans raphé, l'aire axiale est en général conforme à la description de Manguin (1954); cependant, au niveau de l'aire centrale, on note parfois la présence d'une strie raccourcie de chaque côté (fig. 165).

Comme nous l'avons vu précédemment, LangeBertalot et Ruppel (1980) ont montré l'extrême variabilité d'A. minutissima du point de vue morphologique et du point de vue dimensions (4-40 ${ }_{\mu} \mathrm{m} \times 2-4,5 \mu \mathrm{m}$ ). Germain (1981) signale également des formes de passage entre A. microcephala et A. minutissima tout en conservant les deux espèces. Achnanthes modesta (Fig. 206/207) présente des analogies évidentes avec les figures 287 et 288 de A. minutissima dans Lange-Bertalot et Ruppel (1980) et méme avec la figure 43 dans Schœman et Ashton (1982): de même, la disposition des pores (fig. 208) à l'intérieur des cribles semble pratiquement identique à celle de la figure 311 dans Lange-Bertalot et Ruppel (1980). Aux îles Kerguelen, les formes à valves resserrées subcapitées sont aisément reconnaissables au microscope photonique (fig. $70,72,74)$; toutefois, comme nous l'avons déjà montré, elles sont pratiquement identiques à la figure 287 dans Lange-Bertalot et Ruppel (1980). Sans doute serait-il plus logique de ranger cette espèce avec Achnanthes minutissima.

- Achnanthes pseudoaffinis Maillard var. capitata Maillard (fig. 27).

Espèce décrite en Nouvelle-Calédonie (1978). Les deux valves ont une structure identique.

14TH, 23MA (A.C.) ; 4/19/25TH (R.) ; 3DA (R.) ; 20TH (présent).

- Achnanthes pseudolanceolata Hust. (Fig. 75 à 77. 166 à 171, 209 à 211).

Espèce décrite par Hustedt aux iles Kerguelen (1952), retrouvée par Cholnoky (1959) en Afrique du Sud et par Pierre (1977) aux îles Crozet. Il faut 
remarquer qu'une autre espèce, de NouvelleCalédonie, a été décrite par Manguin (1962) sous le même nom.

20/25TH, 112MA (A.C.); 19TH (A.R.) ; 31/56/59TH, 53MA (R.) ; 3/10DA, 9/14/16/38/42TH, 80/94/123MA (T.R.) ; 7/17TH, 24MA (présent); 135MA (T.R.) ; 138MA (R.); 139/140MA (A.C.).

Sur tous les individus observés (tout au moins dans les lacs), le nombre de stries est toujours inférieur à celui donné par Manguin (1954), soit 18-20 au lieu de 24-30 en $10 \mu \mathrm{m}$. De plus, il faut signaler que quelques individus présentent un renflement médian (fig. 166).

Le caractère le plus typique de cette espèce est la présence, sur la valve à raphé, de deux stries nettement plus écartées au niveau de l'aire centrale (fig. 77, 166 à 168) ce qui distingue à coup sûr cette espèce de $A$. lanceolata et de $A$. rostrata Óstrup.

$\mathrm{Au}$ M.E.B. (fig. 166 a 170) et au M.E.T. (Fig. $209 / 210$ ), les stries se résolvent en deux rangées d'aréoles, ce qui distingue aussi nettement cette espèce d'A. lanceolata. Les aréoles sont toujours ouvertes vers l'extérieur. Les cribles se disposent sous forme de rangées parallèles plus ou moins obliques de pores (fig. 211).

Au M.E.B., en vue externe (fig. 168) le raphé apparait souvent plus ou moins flexueux; au niveau de l'aire centrale, les deux branches du raphé s'inflèchissent du même côté et se terminent par des pores étirés ; les fissures terminales s'infléchissent également dans la même direction mais en sens opposé à celui des pores centraux. En vue interne (fig. 167), les stries, délimitant l'aire centrale. présentent une interruption de leur double rangée d'aréoles au voisinage de l'aire axiale : ce caractère est constant chez tous les spécimens observés. Au niveau de l'aire centrale, les deux branches du raphé se terminent par de courts crochets dirigés vers les marges valvaires.

La valve sans raphé, en vue externe (fig. 169), se caractérise par une aire centrale asymétrique marquée par une interruption des stries sur une des marges valvaires (interruption qui coïncide sans doute avec les deux stries plus écartées de la valve à raphé). La face valvaire apparaît bombée de chaque côté de l'aire axiale : celle-ci constitue un sillon médian nettement marqué.

En vue interne (fig. 170/171, 210), le principal caractère est la présence, sur un des côtés de l'aire centrale, d'une sorte de crypte en forme de fer à cheval dont la partie convexe servirait de toit, l'ouverture se trouvant orientée vers le bord de la face valvaire. Ce type de structure est le mème que celui rencontré chez $A$. rostrata Östrup et appelé "cavum" (Moss et Carter 1982). Par ailleurs, lors. que l'attaque à l'acide a été suffisante, toutes les stries présentent une interruption de leur double rangée d'aréoles, côté aire axiale (fig. 170), comme c'est la cas des stries délimitant l'aire centrale sur la valve à raphé.

- Achnanthes pusilla (Grun.) de Toni (in LangeBertalot et Ruppel 1980). 139/140MA (R.).

- Achnanthes rostrata Ostrum (Syn. : A. lanceolata var. rostrata (Öst.) Hust).

Espèce récemment réhabilitée par Moss et Car. ter (1982).

139/140MA (R.).

- Achnanthes saxonica Krasske (fig. 78 à 80,172 à 181, 212 à 214).

Les nombreuses observations de cette espèce, aussi bien dans les lacs que dans les autres milieux, ont montré que l'espèce décrite sous le nom de cocconeis kerguelenensis Manguin (in Bourrelly et Manguin 1954) est en fait Achnanthes saxonica, la confusion étant sans doute due à l'extrême finesse des stries de l'hypovalve.

4/17/33/56TH (R.) ; 5/7/8/24/25/46TH, 111,112MA (T.R.) ; 3/8DA, 10/31TH, 22/49MA (présent) ; 138MA (A.C.) ; 139/140MA (A.R.) ; 135MA (T.R.).

Le M.E.B. (fig. 172,173) confirme la très fine ponctuation des stries de la valve à raphé. En vue interne (fig. 174), le raphé se trouve placé sur une nervure axiale faisant saillie. Le nombre des stries raccourcies délimitant l'aire centrale semble variable, 4 à 6 sur les spécimens des îles Kergue. len, 1 ou 2 sur des exemplaires de Belgique (Leclercq et Fabbri 1982).

Sur la valve sans raphé, en vue externe, les figures $3 \mathrm{~b}$ et $3 \mathrm{c}$ de Leclercq et Fabbri (1982) montrent clairement des stries avec une double rangée de ponctuations; sur les spécimens des îles Kerguelen, cette structure des stries n'est pas toujours évidente (fig. 177, 179, 214). A de forts grossisse. ments, une double rangée de ponctuations réniformes (fig. 180, 214) peut être mise en évidence mais ce n'est pas toujours très régulier. En vue interne, cette double rangée peut être retrouvée 
(fig. 175/176) ou au contraire elle peut disparait re (fig. 178, 181). Cette variabilité peut être due soit à des différences dans le processus de silicification suivant l'origine du frustule ( $f$ ig. 175/176: lac Studer ; 178, 181 : lac des Sternes), soit à des variations dans l'intensité de l'attaque par l'acide.

- Achnanthes stauroneioides Manguin (in Bourrelly et Manguin 1954). (fig. 81 à 83, 182 à 188,215 à 217).

La diagnose doit être légèrement précisée; longueur : 8,5-20 $\mu \mathrm{m}$ au lieu de $12-13 \mu \mathrm{m}$; les stries de la valve sans raphé apparaissent nettement ponctuées au microscope photonique ( $f$ ig. 82/83).

4/8DA (C.); 5DA, 8TH (A.C.) ; 9DA, 4TH (A.R.); 40TH, 29/94MA (R.) ; 6DA, 13/16/19/33TH, 51MA (T.R.) ; 135MA (T.R.) ; 138MA (R.); 139/140MA (A.R.).

En vue externe, au M.E.B. (fig. 182/183) et au M.E.T. (fig. 215), les stries de la valve à raphé sont constituées d'une rangée d'aréoles plus ou moins quadrangulaires ; le raphé est dépourvu de fissure terminale. En vue interne (fig. 184), les terminaisons des deux branches du raphé s'infléchissent vers les marges valvaires au niveau de l'aire cen. trale : au contraire, elles s'infléchissent dans la même direction au niveau de l'hélictoglosse. Sur la valve sans raphé, les aréoles en vue externe (fig. $185,187,216$ ) apparaissent quadrangulaires; en vue interne, elles sont plus grossières (fig. 186, 217) et parfois assez peu visibles (fig. 188). Sur un certain nombre d'individus, on note la présence d'un pore sur la partie polaire de l'aire axiale ( $f \mathrm{~g}$. 216/217).

- Achnanthes stauroneioides forma striata Maillard, nov. fo. (fig. 28).

Diffère de l'espèce par la présence dans l'aire centrale stauroneiforme de stries généralement plus écartées que les autres.

4DA (C.) ; 31TH (A.C.) ; 14TH (R.); 20TH (T.R.).

\section{5. - Conclusion}

1) La plupart des espèces rencontrèes sont cosmopolites : les taxons Cocconeis therezieni, Achnanthes contusa et var. atomoïdes, A. manguinii et var. elliptica, A. stauroneioides et fo. striata, A. abundans et A. modesta peuvent étre considérés comme endémjques des îles Kerguelen. Les deux dernières espèces citées, comme l'ont mont ré les commentaires concernant ces deux taxons, peuvent prêter à discussion. Il semblerait qu'elles puissent s'intégrer dans le spectre de variation de $A$. minutissima, tel que l'ont proposé Lange-Bertalot et Ruppel (1980). Le degré de variabilité d'une espèce de dia tomée est encore (malheureusement ?) laissé libre à l'interprétation de l'observateur. Pour trancher la question, l'idéal serait sans doute d'isoler les différentes variantes d'une même espèce et les espèces qu'on pourrait éventuellement y intégrer, de les cultiver ensuite afin de suivre leur évolution morphologique en fonction de différents milieux de culture. Peutêtre alors serait-on, dans la définition d'une espèce, obligé de faire appel à d'autres critères que la traditionnelle observation du frustule.

2) Chez quelques espèces, A. lapponica var. ninkei (fig. 148/149), A. pseudolanceolata (fig. 169) et $A$. saxonica (fig. 181), on observe la présence d'une gouttière obstruée sur l'aire centrale, gouttière s'interrompant au niveau de l'aire centrale. Selon Simon. sen (1979) et Andrews (1981), cette cannelure représente le vestige du raphé ancestral. Chez d'autres espèces, A. abundans (fig. 108), A. confusa (Fig. 121), A. austriaca (fig. 109, 112), A. manguinii (fig. 153), A. stauroneioides (Fig. 216/217), on observe toujours ou souvent la présence d'un pore (souvent en forme de fente entourée d'une bordure saillante en vue interne) aux extrémités de l'aire axiale. Doit-on interpréter cette présence comme le vestige du pore terminal, ce qui confirmerait les suggestions des deux auteurs précités, ou, au contraire, doit-on y voir le début d'un processus inverse. Dans ce cas, on pourrait interpréter cette "st ructure " comme une étape de la transformation d'un processus labié en raphé. Si cette hypothèse s'avérait exacte, l'évolution pourrait ou aurait pu se faire dans les deux sens : biraphidées $2=1$ monoraphidées. Schrader (1974) pense que les monoraphidées representent le stade primitif du système raphéen plus compliqué des biraphidées, ce qui serait "pro parten en accord avec l'hypothèse 2 . La question se pose de savoir pourquoi les deux valves n'ont pas évolué ou n'évoluent pas parallèlement. Le mode de vie peut éventuellement apporter un semblant de réponse, comme cela a déjà été proposé. Aux îles Kerguelen, étant donné l'isolement du milieu, l'examen des sédiments pourrait peut-ètre permettre de résoudre le probleme.Line remarque en conclusion: chet A. confusa, le pore "terminal" sur la valve sans raphé est toujours présent alors qu'il est toujours absent chez $A$. confusa var, atomoides. Si l'on admet l'hypothese 1, la variété est plus évoluée que l'espèce; il serait donc plus logique de faire monter la variété au rang d'espece et de rétrograder l'espèce au niveau de variété 

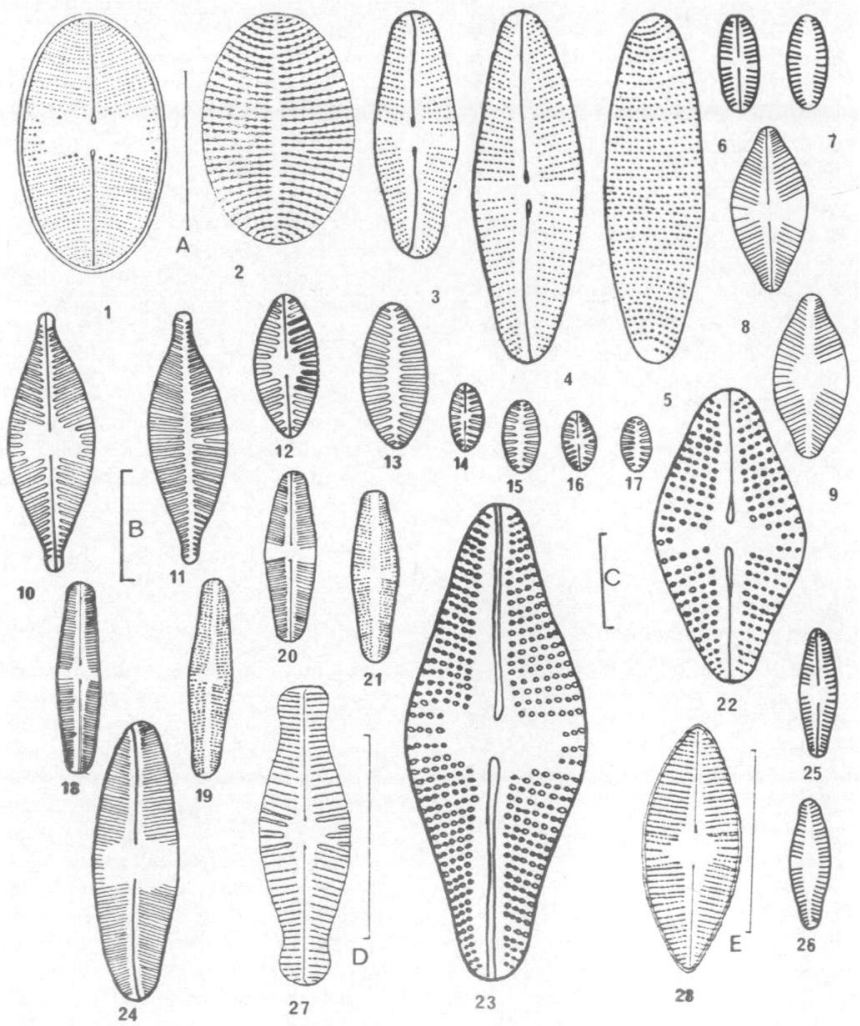

\section{PLanche}

1 : Cocconeis therezieni nov. sp. (valve à raphé : 2 : $\mathrm{C}$. therezieni nov. sp. (valve sans raphé); $3 / 4 / 5:$ Achnanthes coarctata var. elliptica; $6 / 7: A$. conspicua var, brevistriata : 8/9:A. germainii ; $10 / 11:$ A. delicatula ss sp. delicalula; 12/13: A. delicatula ss sp. hauckiana; 14/15/16/17: A. engelbrechtii ?; 18/19/20/21: A. confusa;
22/23: A. inflata ; 24 : A. lapponica var, ninkei (Syn. : A. aretasii) ; 25/26:A. gibberula; $27: A$. pseudoaffinis var. capitata ; 28 : A. stauroneioides fo. striata nov. fo. Fig, $1 / 2$ : échelle A ; Fig. 3/4/5/8/9/10/11/22/23/24 : échelle B. Fig. $6 / 7 / 12 / 13 / 14 / 15 / 16 / 17 / 18 / 19 / 20 / 21 / 25 / 26$ : échelle $C$; Fig. 27 ; échelle D; Fig. 28 : échelle E. 
(13)

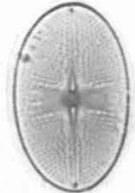

29

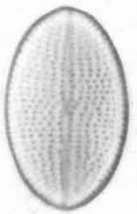

34
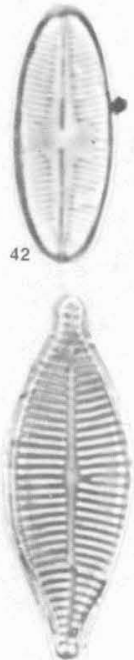

47

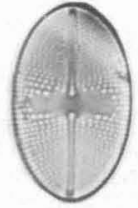

30
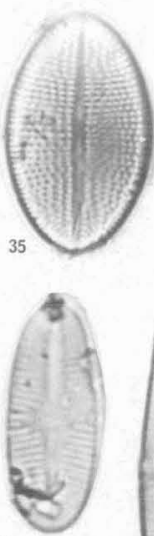

43

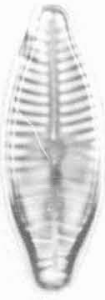

48

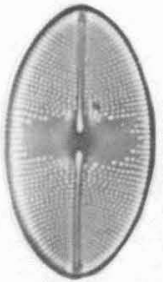

31
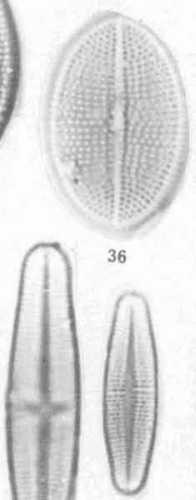

45

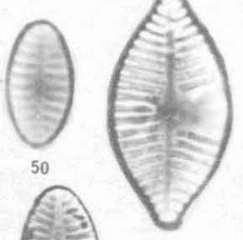

52

49

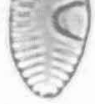

51

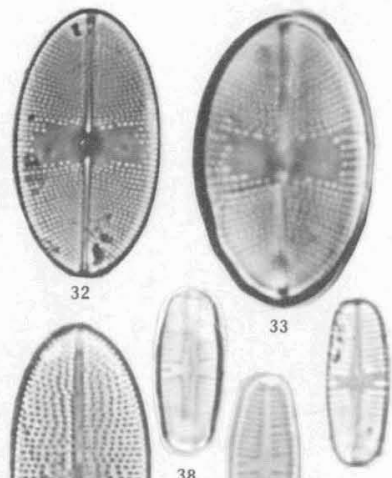

40

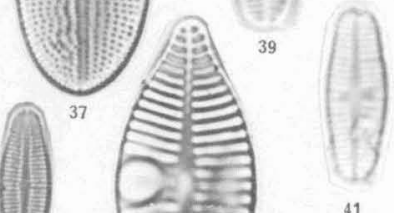

41

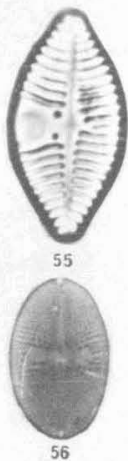

confusa $(\times 2700): 45 / 46:$ A. confusa var. atomoides $(\times 2700): 47 / 48:$ A. delicatula ss. spec. delicatula $(\times 2000) ; 49 / 50:$ A. engelbrechtii ? $(\times 2700) ; 51:$ A. lanceolata $(\times 2000) ; 52 / 53 / 54 / 55:$ A. lanceolata var. lanceo-

\begin{abstract}
latoides $(\times 2000) ; 56$ : Cocconeis therezieni $(\times 2000)$.
\end{abstract}
Planche II (photos en microscopie photonique).

29/30/31/32/33: Cocconeis therezieni nov. sp., valve à raphé $(\times 2000) ; 34 / 35 / 36 / 37$ : Cocconeis therezieni nov. sp., valve sans raphé $(\times 2000) ; 38 / 39 / 40 / 41$ : Achnanthes abundans $(\times 2700) ; 42 / 43: A$ austriaca $(\times 2700) ; 44: A$. 


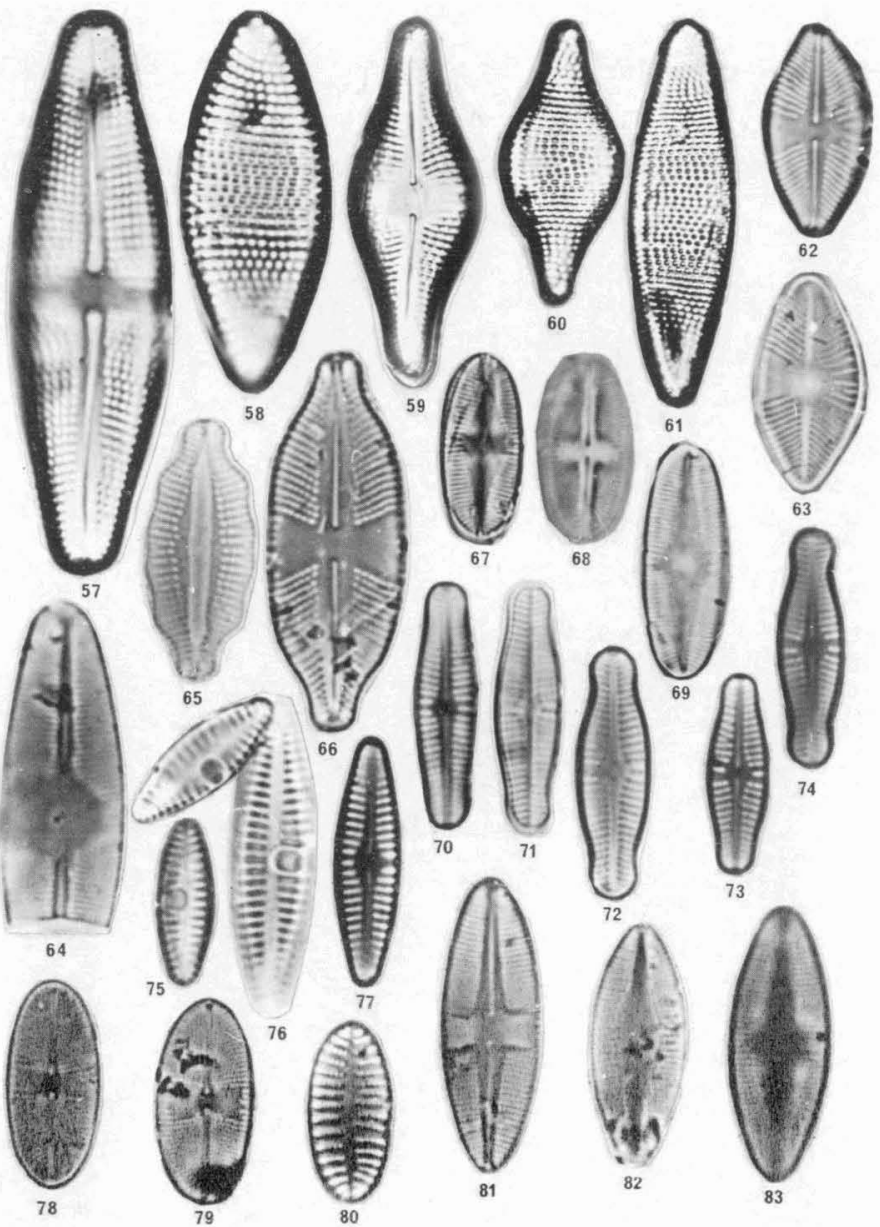

Planche III (photos en microscopie photonique).

57 : Achnanthes coarctata ( $\times$ 2000); $58:$ A. coarctata var. elliptica $\{\times 2000)$; 59/60/61: A. inflata $(\times 1000)$; $62 / 63 / 64:$ A. lapponica var. ninkei $(\times 2700) ; 65:$ A. manguinit $(\times 2700): 66:$ A. manguinii var. elliptica $(\times 2700)$; 67/68: A. marginulaı, les deux valves du même frustule $(\times 2000) ; 69: A$. marginulata $(\times 2000) ; 70 / 71: A$.

modesta ( $\times 2700)$; 75/76: Achnanthes pseudolanceolata, vues internes de la valve sans raphé $(\times 2700) ; 77: A$. pseudolanceolata, valve à raphé $(\times 2700) ; 78 / 79 / 80: A$. saxonica $(\times 2000) ; 81 / 82:$ A. stauroneioides $(\times 2700) ; 83$ : A. stauroneioides, vue interne de la valve sans raphe $(\times 2700)$. 


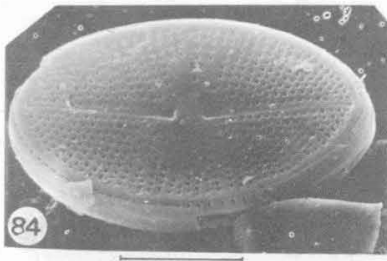

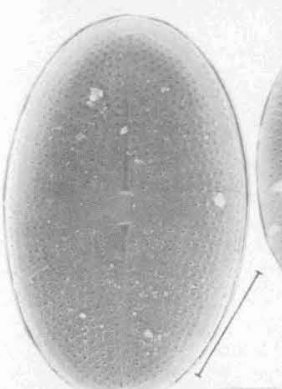
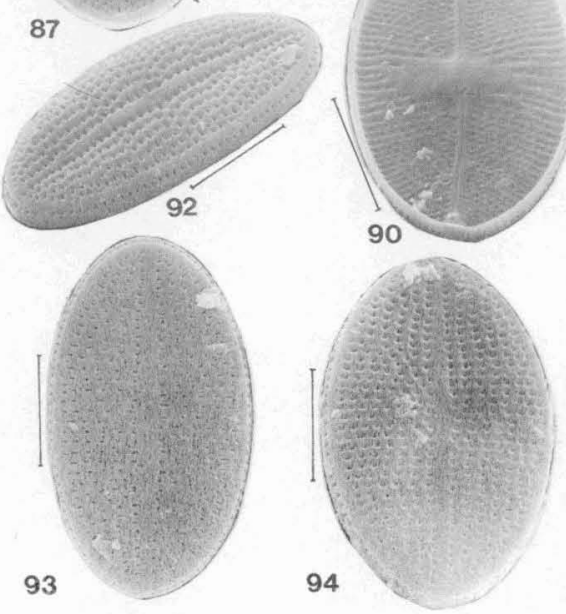
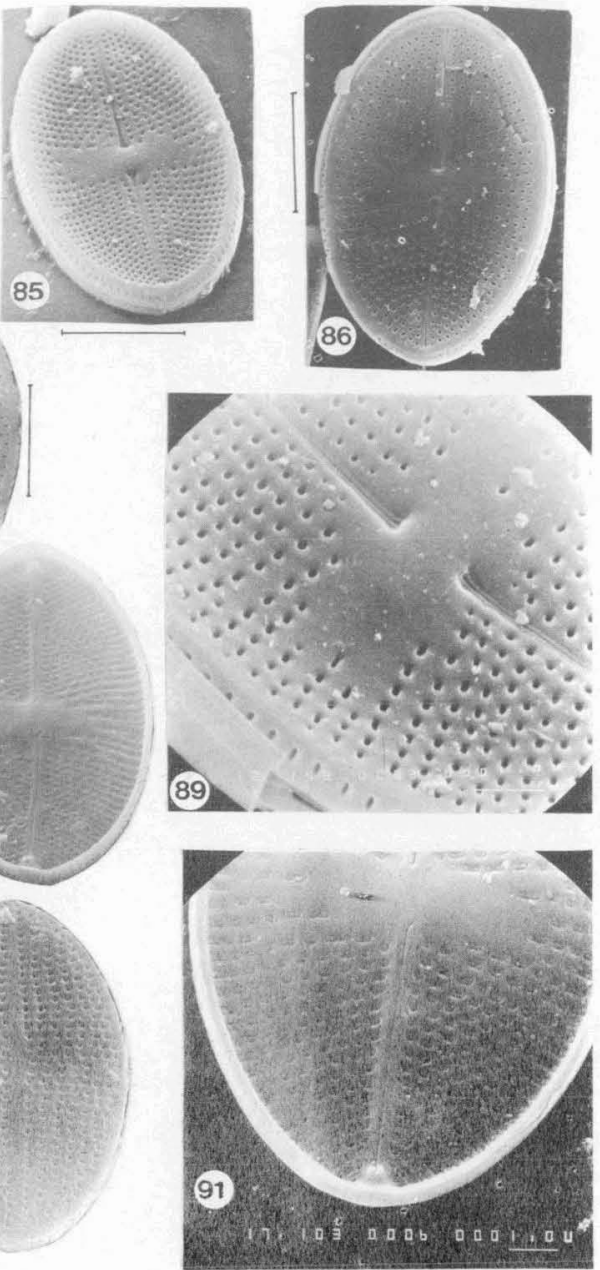

Planche iv (photos en microscopie électronique à balayage).

Cocconeis therezeini nov, sp. ; $84 / 86$ : valve à raphè du même individu, vue externe ; $85 / 87$ : valve à raphé du même individu, vue externe ; 88 ; valve à raphé ; 89 ; cen- tre de la figure 84 a un fort grossissement ( $\times 15000)$ : $90 / 91$ : valve à raphé, vue interne : $92 / 93 / 94$ : valve sans raphé. vue externe.

Le trait de l'échelle indique $5 \mu \mathrm{m}$ sauf Fig. 89 et $91,1 \mu \mathrm{m}$. 


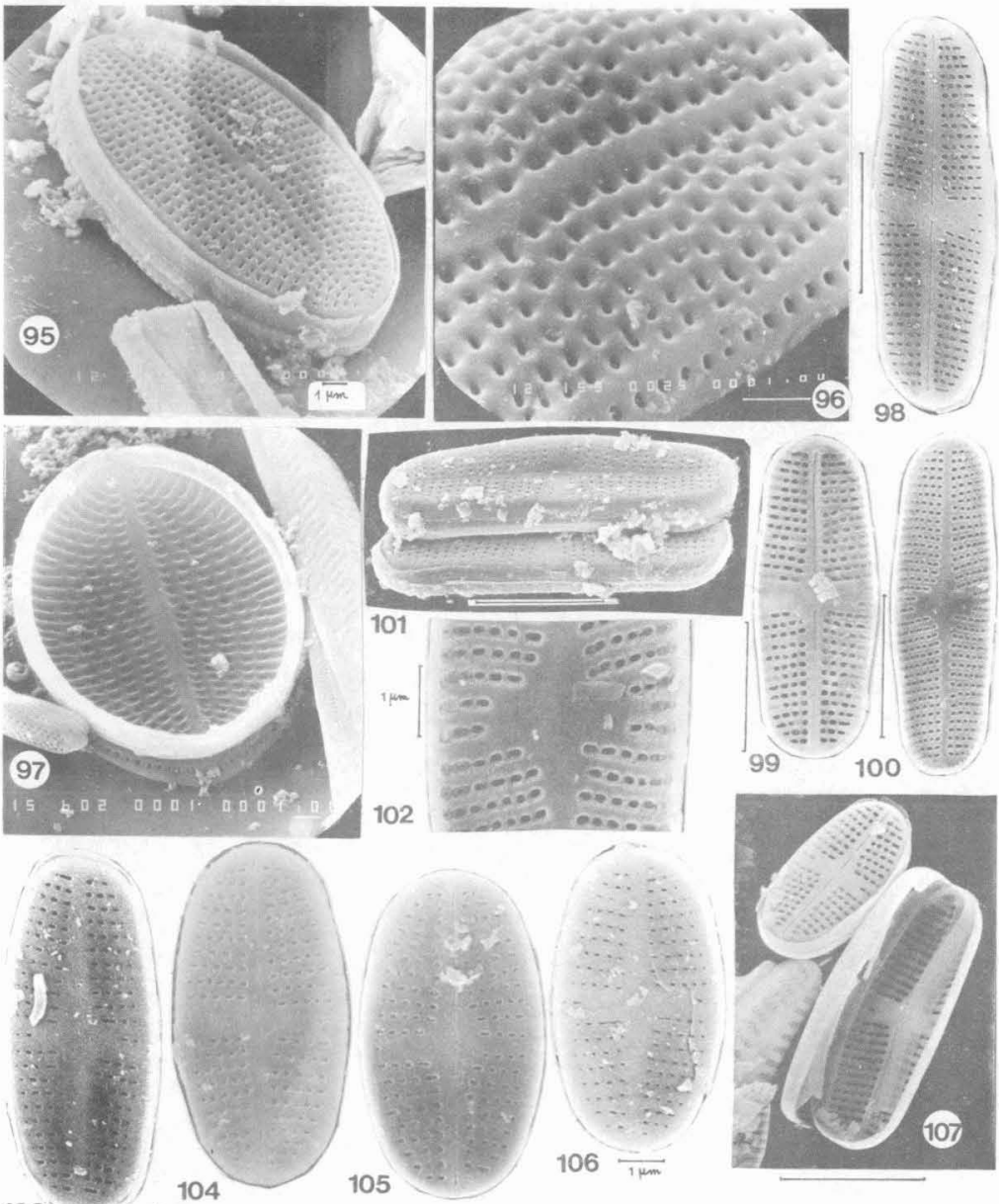

103

Planche v (photos en microscopie électronique à balayage).

Cocconeis therezieni : 95 : valve sans raphé, vue externe, bande connective : 96 ; valve sans raphé depourvue de bande connective, méme individu que Fig. 92, détail du centre : Achnanthes abundans : $98 / 101 / 104 / 105:$ valve à raphé, vue externe ; 99 : valve à raphé, vue interne : 100 :

valve sans raphé, vue interne : 102 : centre du méme individu à un fort grossissement ; 103/106: valve à raphé, vue externe : 107 : vue interne et externe de la valve à raphé.

Le trait de l'échelle indique $5 \mu^{\mathrm{m}}$ sauf Fig. 95/96/97/102/106, ${ }_{\mu}{ }_{\mu} \mathrm{m}$. 

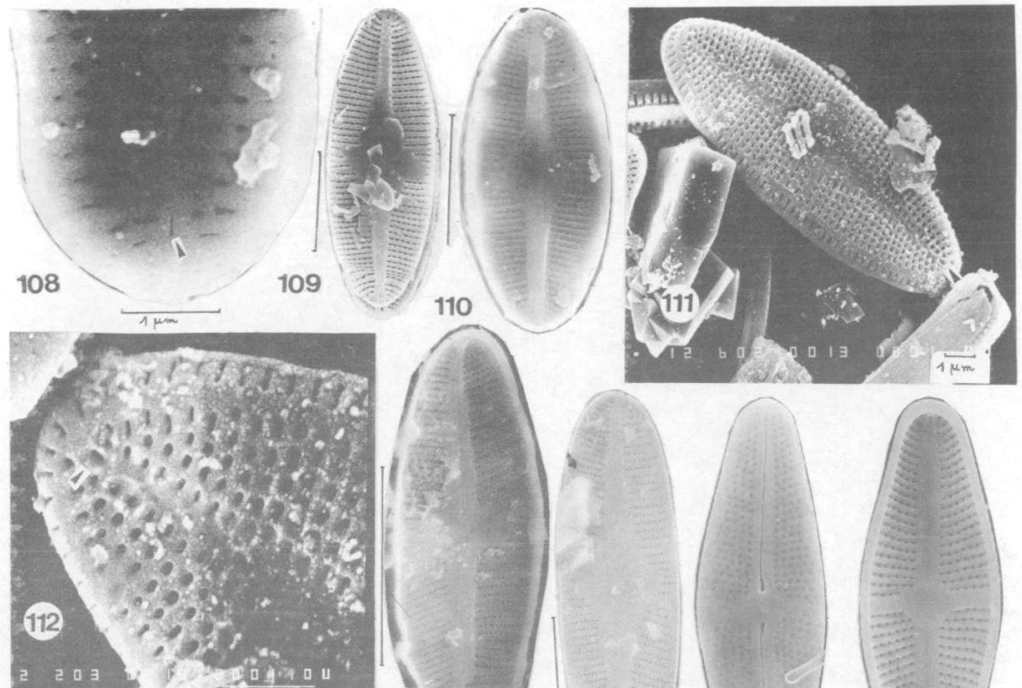

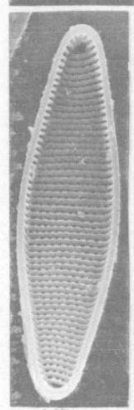

117

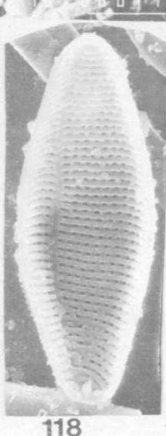

119

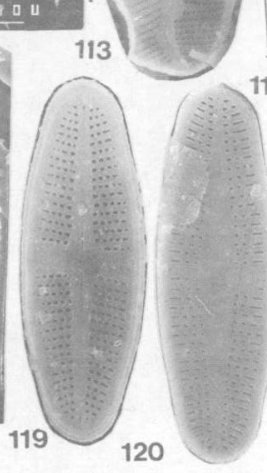

114

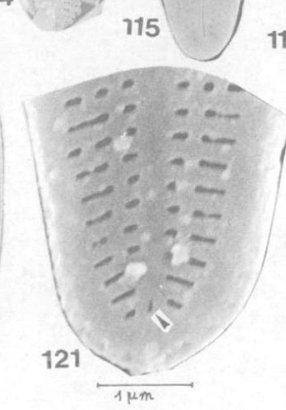

122
Planche vi (photos en microscopie électronique a balayage).

108: Achnanthes abundans, pore à l'extrémité de l'aire axiale à un fort grossissement, vue interne (flèche) : $A$ ch. nanthes austriaca; 109 ; valve sans raphé, vue externe : un pore à chaque extrémité de l'aire axiale : 110 : valve sans raphé, vue interne; 111 : valve sans raphé, vue externe, pore à chaque extrémité de l'aire axiale (flèche) : 112 : pôle du mème spécimen à un fort grossissement : Achanthes austriaca var. helvetica: 113 : value à raphé, vue inteme : 114 : valve sans raphé, vue externe: Ach. nanthes coarctata var elliptica: 115 : valve à raphé, vue externe : 116 : valve à raphé, vue interne : 117 : valve sans raphé. vue interne, présence d'un worbiculus * aux deux extrémités ; 118 : valve sans raphé, vue externe. Achnan. thes confusa : 119 : valve à raphé, vue externe : 120 : valve sans raphé, vue externe, un pore à chaque extrémité de l'aire axiale : 121 : pôle à un fort grossissement, pore à l'extrémité de l'aire axiale (flèche), vue externe; 122 : Achnamhes confusa var. atomoides, vue externe. Le trait de leechelle indique $5 \mu \mathrm{m}$ sauf Fig. $108 / 111 / 112 / 121,1 \mu$. 

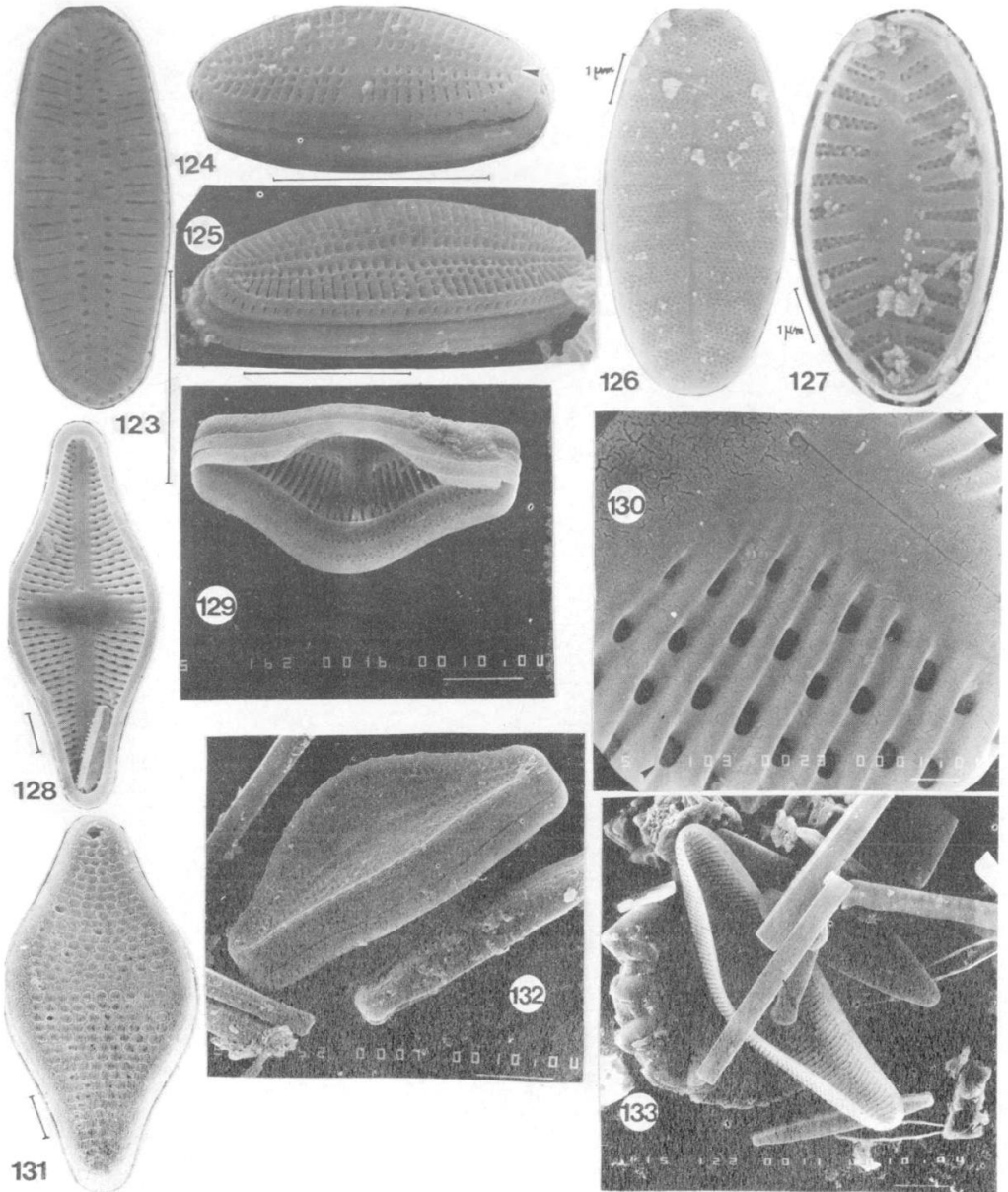

Planche vil (photos en microscopie électronique à balayagel.

123: Achnanthes confusa var. atomoides, valve sans raphé, vue externe; 124 : Achnanthes confusa, valve sans raphé, vue externe, un pore aux extrémités de l'aire axiale (flèche); $125:$ Achnanthes confusa var. atomoides, valve sans raphé, vue externe : 126 : Achnanthes delicatula ss. sp. hauckiana, valve sans raphe, vue externe: 127: Achnanthes engelbrechtii, valve sans raphé, vue interne; Achnanthes inflata; 128 : valve à

raphé, vue interne ; 129 : valve à raphé avec bande connective ; 130 : vue interne de la valve à raphé a un fort grossissement, terminaison centrale d'une branche du raphé et velum (fleche): 131: valve sans raphé, vue externe, a orbiculus n visible à l'extrémité supérieure : 132 : valve sans raphé, vue externe: 133 : valve sans raphé, vue interne, pseudo-raphé visible (flèche).

Le trait de l'échelle indique $5 \mu \mathrm{m}$ sauf Fig. 126/127/130.

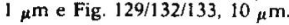



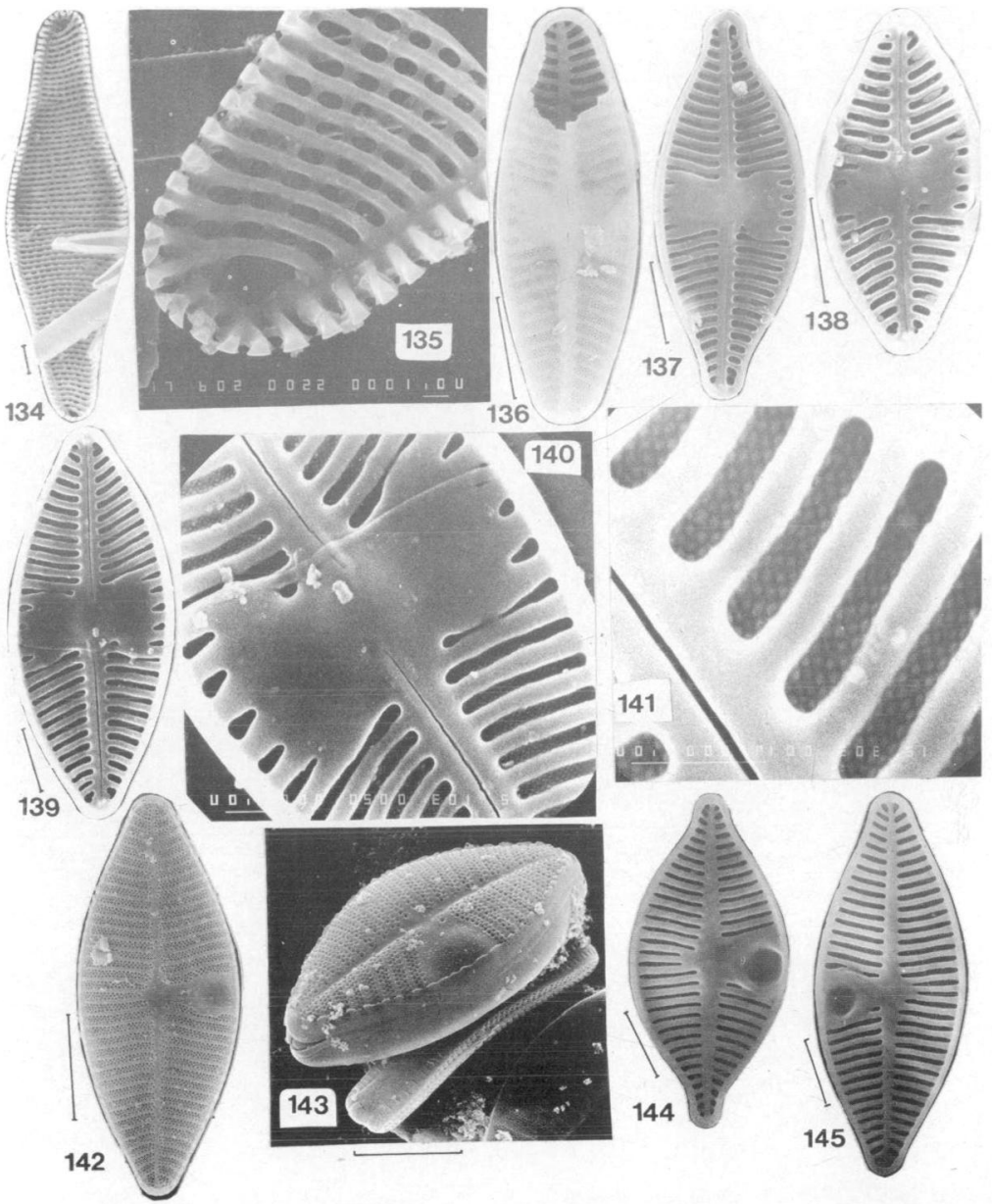

Planche viIt (photos en microscopie électronique à balayage).

Achnanthes inflata : 134 : valve sans raphè, vue interne, *orbiculus n aux deux pôles : 135 : póle du même spécimen à un fort grossissement, terminaison du pseudo. raphe sur la partie droite; 136 : Achnanthes lanceolata, valve à raphé, vue externe; Achnanthes lanceolata var. lanceolatoides ; $137 / 138 / 139$ : valve à raphé, vue interne montrant les différentes structures des stries de l'aire centrale ; 140 : centre du spécimen précédent à un fort grossissement ; 141 : vue interne des stries à fort grossissement ; 142 : valve sans raphé, vue externe: 143 : valve sans raphé, vue externe, bande connective; 144/145 : valve sans raphé, vue interne.

Le trait de l'échelle indique $5 \mu \mathrm{m}$ sauf Fig. 135/140/141, $1 \mu \mathrm{m}$. 

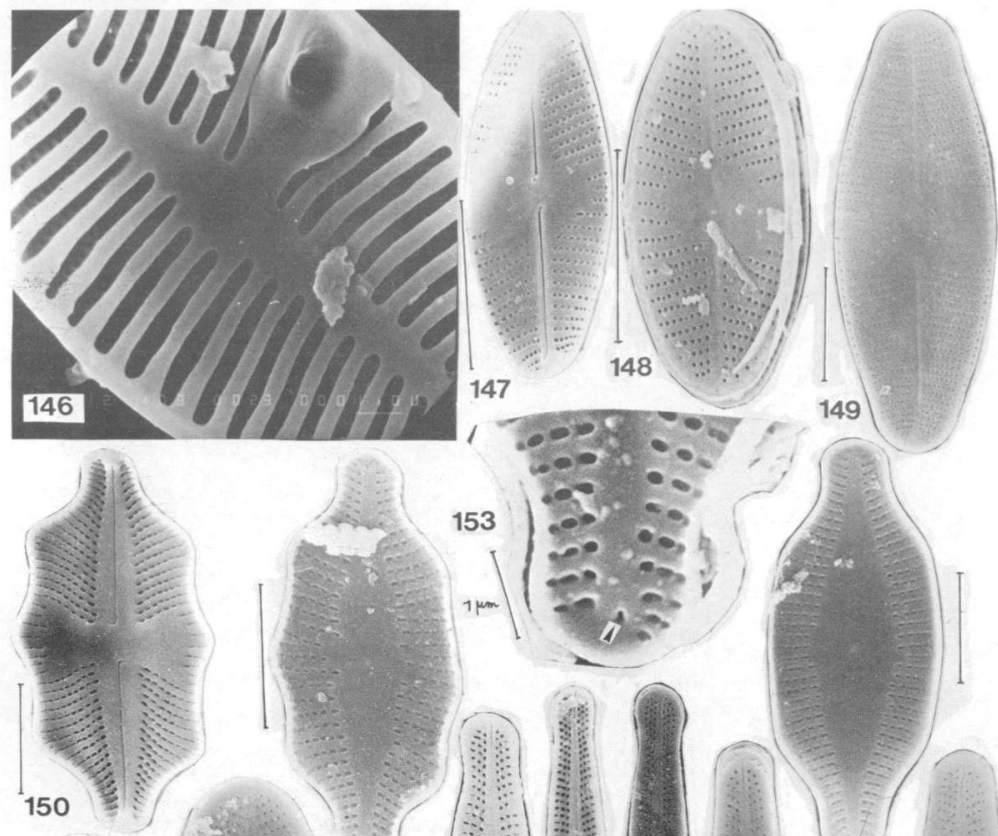

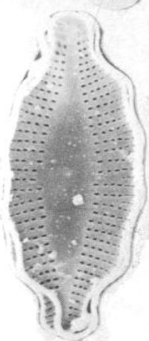

152

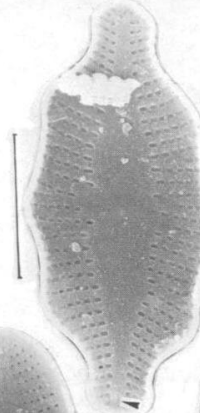

151

Planche IX (photos en microscopie électronique à balayage).

146 : Achnanthes lanceolata var. lanceolatoides, "sinus" à un fort grossissement, vue interne: Achnanthes lapponica var, ninkei: 147 : valve à raphé, vue interne : 148 : valve sans raphé, vue externe; 149 : valve sans raphé, type aretasii, vue externe : Achnanthes manguinii : 150 : valve à raphé, vue interne ; 151 : valve sans raphé, vue interne, pore aux deux extrémités de l'aire axiale (fleche) ; 152 : valve sans raphé, vue interne, pore

153

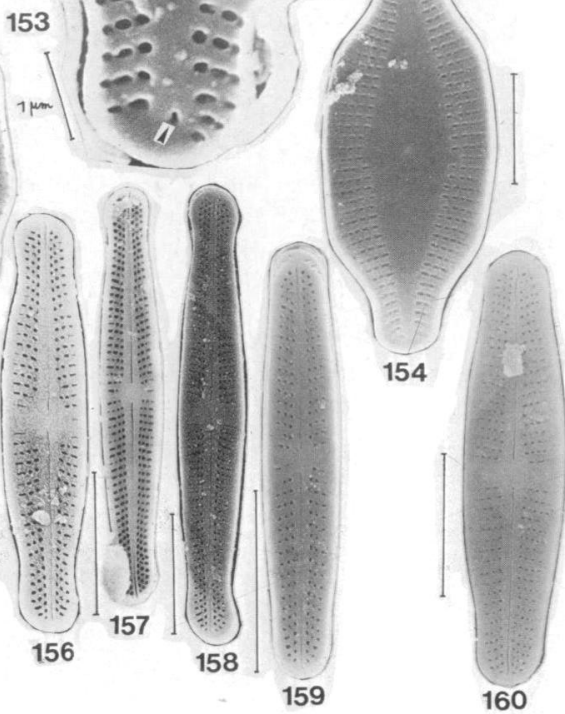

visible à l'extrémité supérieure de l'aire axiale, valvocopula décollée : 153 : même individu, pore à l'extrémité de l'aire axiale à un fort grossissement ; 154 : Achmanthes manguinii var, elliptica, valve sans raphé, vue interne : 155: Achnanthes marginulata, vue externe; 156/157/158/159: Achnanthes minutissima, valve à raphé, vues externes et internes; 160 : Achnanthes confusa.

Le trait de l'échelle indique $5 \mu \mathrm{m}$ sauf Fig. 146/153, $1 \mu \mathrm{m}$. 

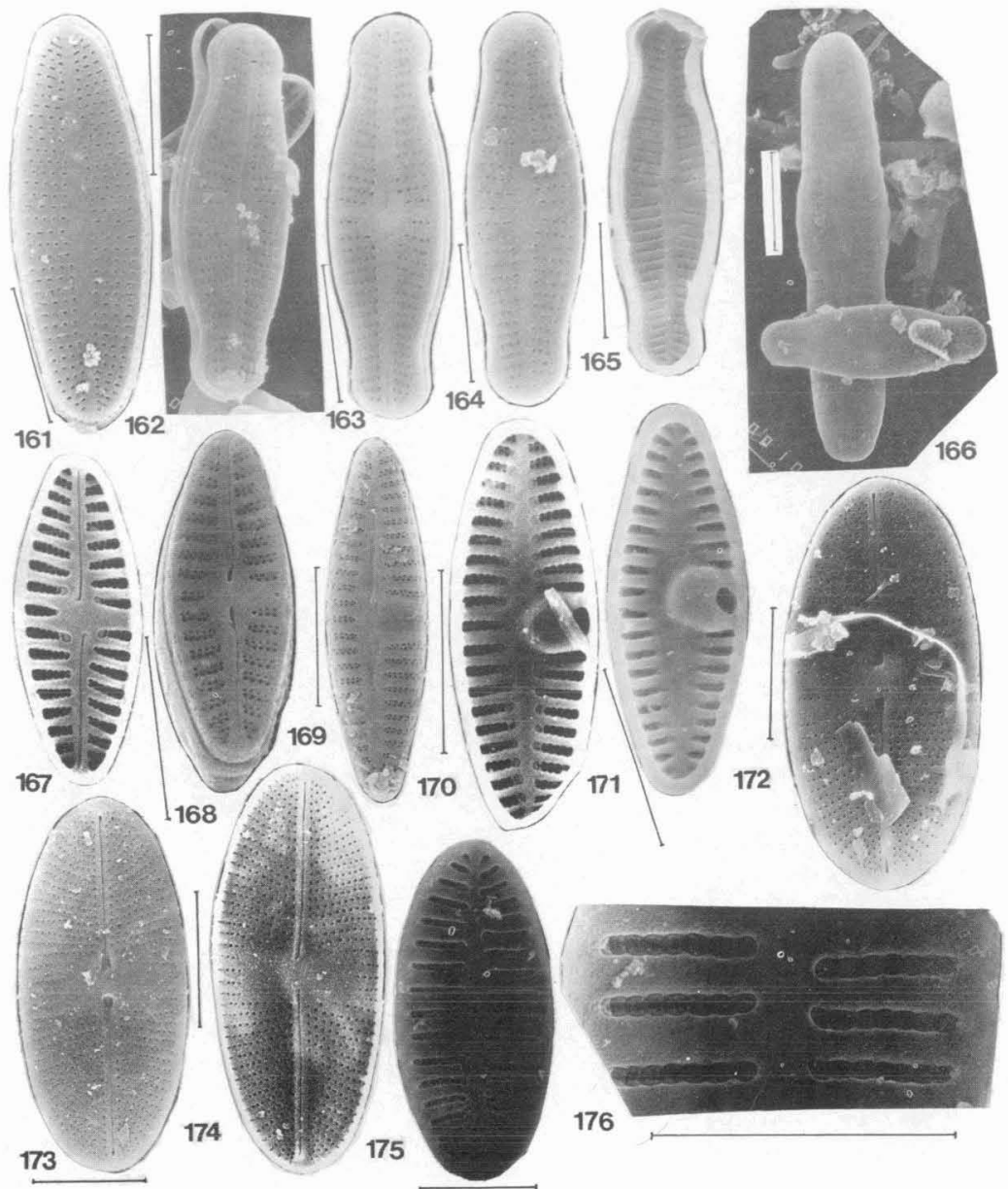

176

Planche $x$ (photos en microscopie électronique à balayage).

161 ; Achnanthes minutissima: Achnanthes modesta: $162 / 163$ : valve à raphé, vue externe; 164 : valve sans raphé, vue externe ; 165 : valve sans raphé, vue interne. Achnanthes pseudolanceolata; 166 : valve a raphé. vue externe, variation de la forme : 167 : valve à raphe, vue interne, forme typique: 168: valve à raphé, vue

externe ; 169 ; valve sans raphe, vue externe ; 170 ; valve sans raphé, vue interne; 171 : valve sans raphé, vue interne, "cavum": Achnanthes saxonica: 172/173: valve à raphé, vue externe: 174 : valve à raphé, vue interne : 175 : valve sans raphé, vue interne ; 176 : même individu que le précèdent, stries à fort grossissement.

Le trail de l'échelle indique $5 \mu$. 

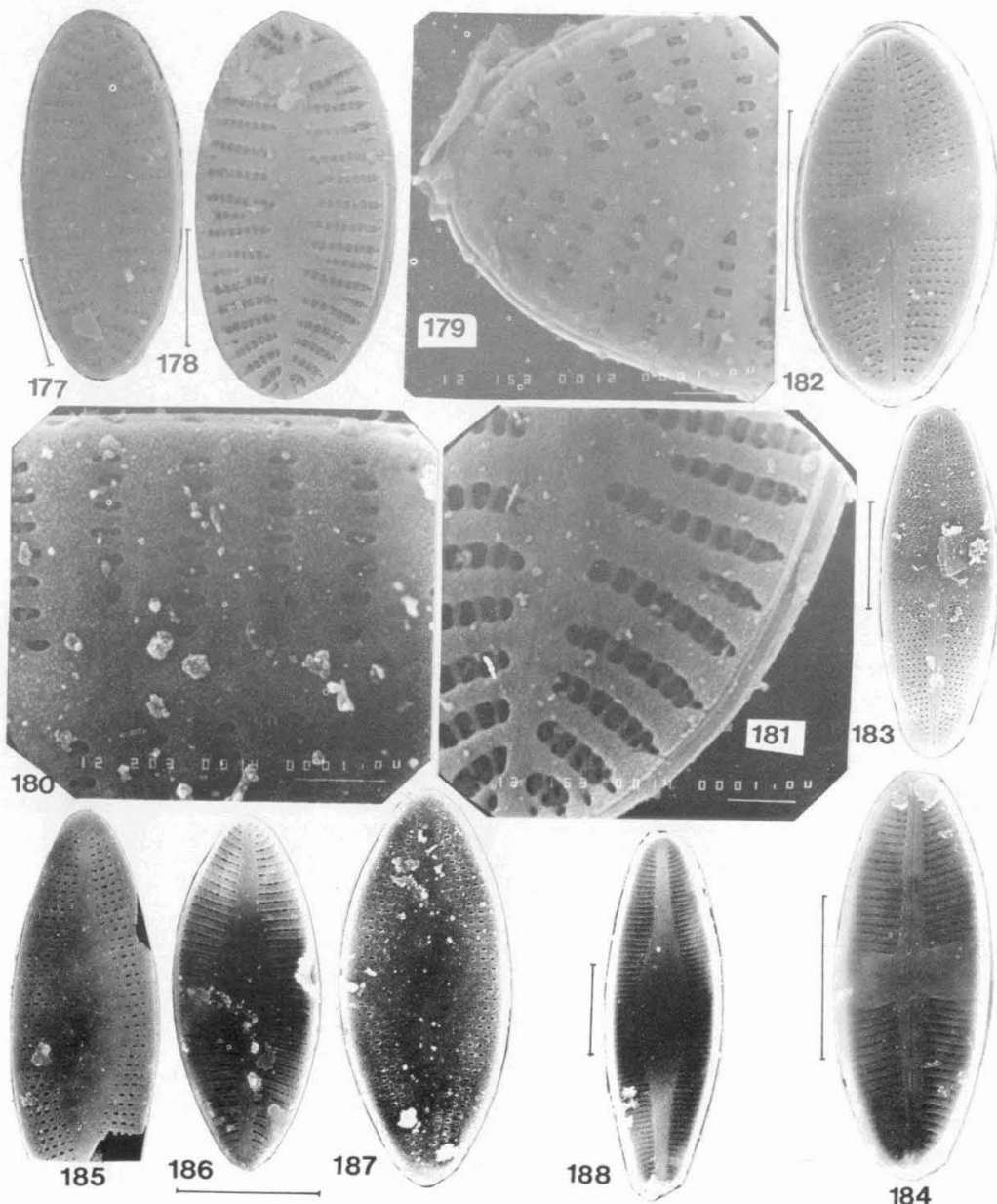

Planche Xi (photos en microscopie électronique à balayage).

Achnanthes saxonica, valve sans raphé; 177: vue externe; $178:$ vue interne $; 179 / 180$ : vue externe à fort grossissement, 181 : vue interne à fort grossissement ;
Achnanthes stauroneioides ; $182 / 183$; valve à raphé, vue externe ; 184 : valve à raphé, vue interne ; $185 / 187$ : valve sans raphé, vue externe ; $186 / 188$ : vue interne.

Le trait de l'échelle indique $5 \mu \mathrm{m}$ sauf Fig. 179/180/181, $1 \mu \mathrm{m}$. 

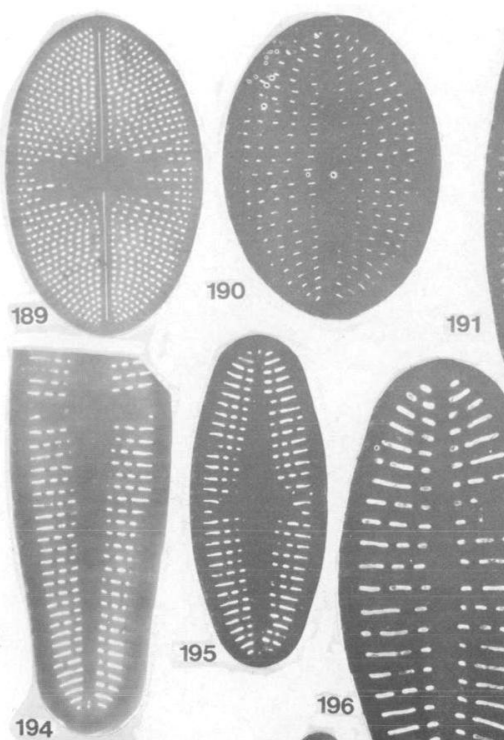

191
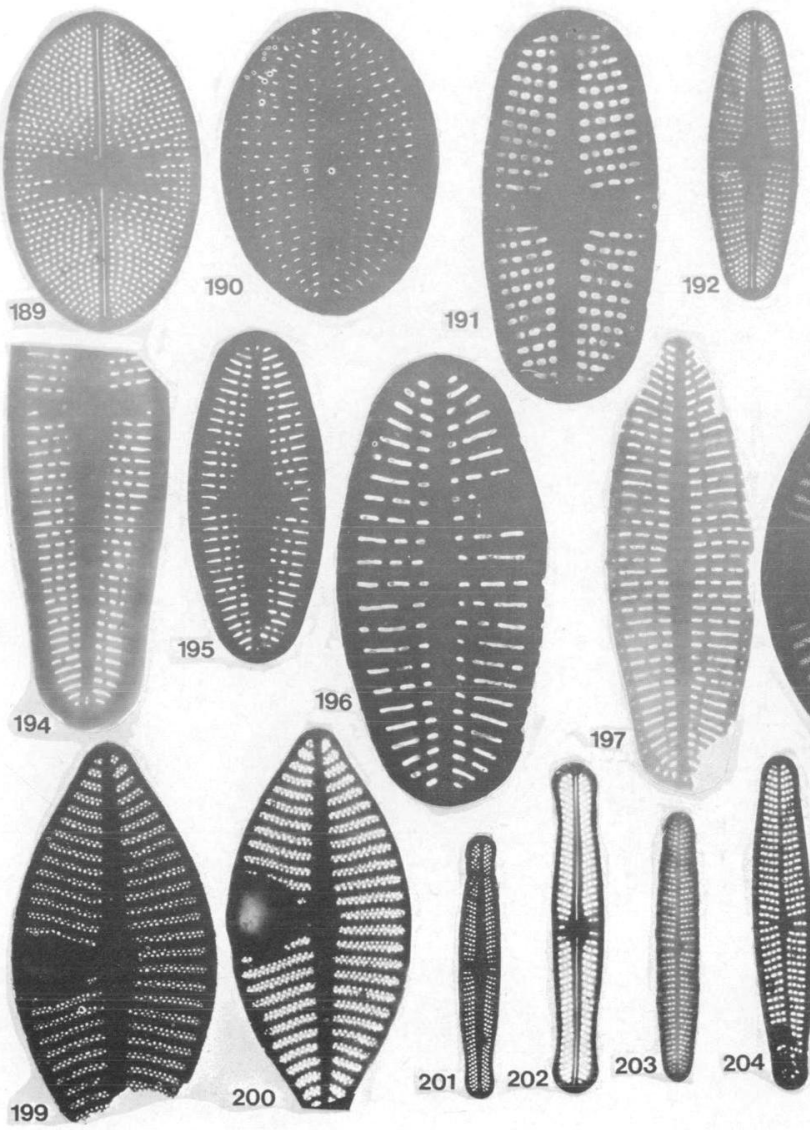

Planche xll (photos en microscopie électronique à transmission).

Cocconeis therezieni : 189 : valve à raphé \#2600; 190 : valve sans raphé \#3300; 191 ; Achnanthes abundans \#6600; Achnanthes confusa: 192: valve à raphé \# 3300 ; 193 : valve sans raphé \# $3300 ; 194$ : valve sans raphé, vue interne, pore à l'extrémité de l'aire axiale, \# 8300 ; 195 : valve sans raphé avec pore visible à l'ex-

trémité de l'aire axiale \# 5000 ; Achnanthes confusa var. atomoïdes : 196 : valve sans raphé \# 9000 : 197 : valve sans raphé \# 5000 ; Achnanthes lanceolata var. lanceolatoïdes: 198 : valve à raphé, 12000 ?; 199 : valve sans raphé, vue externe \# $3300: 200$ : valve sans raphé, vue interne, 9000 ?; Achnanthes minutissima ; 201 : valve à raphe $\# 2600 ; 202: \# 3300: 203$ : valve sans raphé \# $2600: 204: \# 3300 ; 205$ : valve à raphé, vue interne, \#5000. 

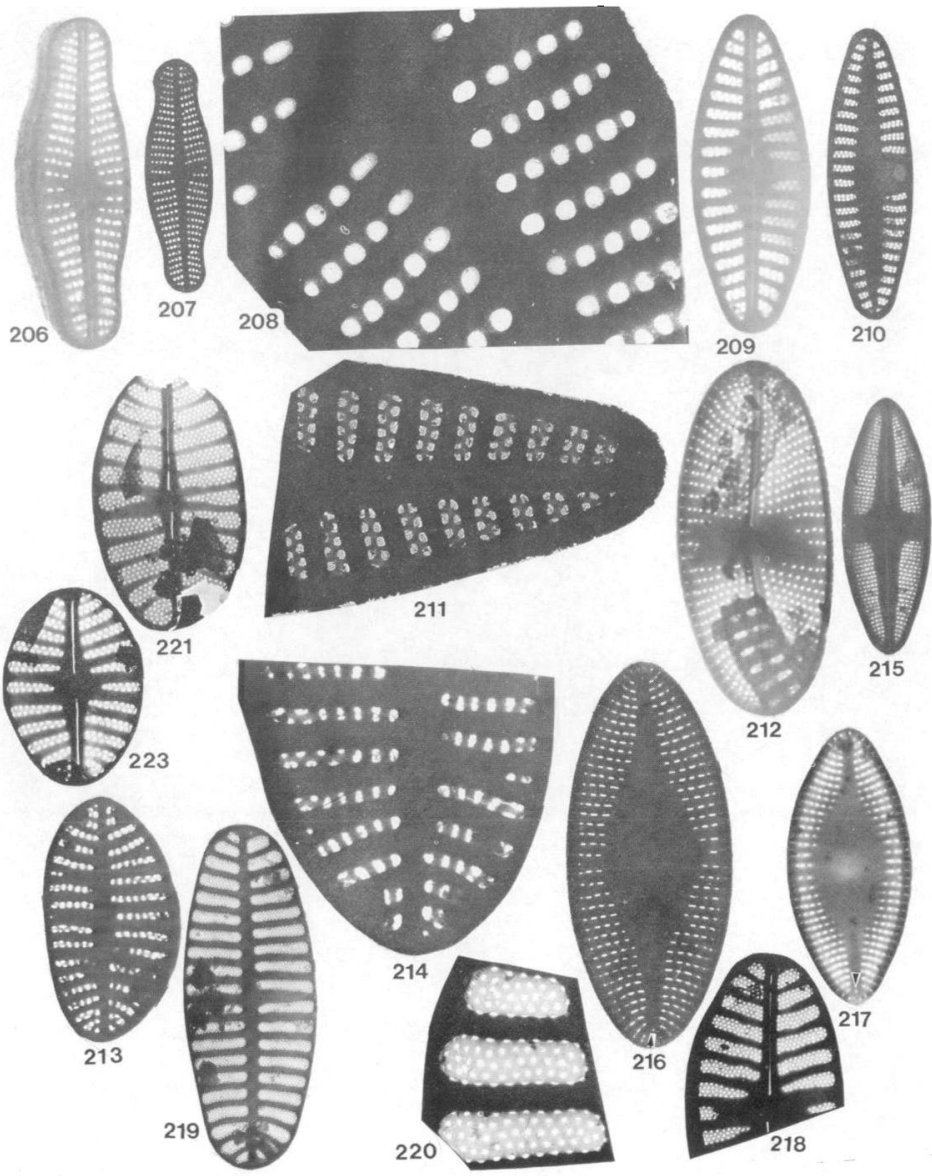

Planche XIII (photos en microscopie electronique a transmission).

Achnanthes modesta ; 206 : valve à raphé \#5000: 207 : valve sans raphé $2600: 208$ : détail des stries, cribles \#16000; Achnanthes pseudolanceolata: 209 : valve à raphé \#3300:210: valve sans raphé, vue interne \# $3300 ; 211$ : valve sans raphé, détail des stries, cribles \#13300: Achnamthes saxonica: 212 : valve à raphé \# $3300 ; 213$ : valve sans raphé \#2600; 214 : mêrne individu

\#6000; Achnanthes stauroneioides; 215 : valve à raphé \# 2000: 216/217: valve sans raphé, vue externe et vue interne, pore à l'extrémité de l'aire axiale (flèche), \# 5000 et 3300 ; Achnamihes delicarula ssp. hauckiana: 218 et 221 : valve à raphé \#6000; 219 : valve sans raphé \# 4000: 220 : détail des stries, même individu \# 20000 ; Achnanthes engelbrechtii, 223, valve à raphé, 2 rangées d'aréoles par strie. \#6000. 


\section{Travaux cltés}

Andrews (G.W.). 1981.- Achmanthes linkei and the origin of mono raphid Diatoms. Bacillaria, $4: 29-40$.

Bourrelly (P.) \& Manguin (E.). 1954,- Contribution à la flore algale d'eau douce des iles Kerguelen. Mém. Inst. Sc. Madagascar. sér. $B, 5: 7.58$.

Camburn (K.E.), Lowe (R.L.) \& Stonebumer (D.L.). 1978 - The hap tobenthic diatom flora of Long Branch Creek, South Carolina. Nova Hedwigia, XXX: 149-278.

Carter (J.R.). 1966.- Some fresh water diatoms of Tristan de Cunha and Gough island. Nova hedwigra, XI (1/4): 443-483. pl. 60/68.

Carter (J.R.) \& Denny (P.). 1982. - Freshwater algae of Sierm Leone IV Bacillariophyceae : Part I : Diatoms form the river Jong (Taia) at Njala. Nova Hedwigia, $73: 281-231$.

Cholnoky (B.J.). 1959. - Neue und settene Diatomeen aus Afrika. IV. Diatomeen aus der Kaap-Provinz. Osterr. Botart. Zeirsch rift. $106: 1.69$.

Coste (M.1. 1978.- Sur l'utilisation des diatomées benthiques pour l'appréciation de la qualité biologique des eaux courantes. Thèse Univ, Besançon: 143 p., $11 \mathrm{pl}$.

Coste (M.) \& Ricard (M.). 1982. - Contribution à l'étude des diatomées d'eau douce des Seychelles et de l'íle Maurice. Crvpiogamie : Algologie, III (4): 279.313

Gerloff (J.) \& Helmcke (J.G.). 1977. - Diatomeenschalen im Elekironenmikroskopischen Bild, Teil $X$, Cramer, Vaduz: pl $924 / 1023$

Germain (H.). 1937. - Diatomées d'une tourbe des îles Kerguelen Bull. Soc. Fr. Microsc., VI (1):11-17.

Germain (H.). 1981. - Flore des Diatomees, eaux douces et saumátres. Boubé, Paris : 444 p.

Germain (H) et Le Cohu (R.). 1981 - Variability of some features in a few species of Gomphonema from France and the Kergue. Ien Islands (South Indian Ocean). Proc. 6th Simp. Recem and Fossil Diatoms, Budapest 1980, Kaitz, Kanigstein : 167.178.

Guemeur (P.) \& Manguin (E.). 1953. - Notes sur quelques diatomès rares ou nouvelles. Osterretch. Bot. Zeitschr., $100(4 / 5)$ : 541.548.

Hustedt (F.). 1937/38. Systematische und Okologische Untersuchungen uber die Diatomeenflora von Java. Bali und SumatraSystematischer Teil. Archiv. Hydrobiol., Suppl. 15/16:131/177 : $187-295: 393-506$

Hustedt (F.). 1952. - Neue und wenig bekannte Diatomeen. Särryk Boraniska Not. 4: $366-410$.

Hustedt (F.) 1959.- Die Kieselagen. In : Kryptogamen-Flora von Deuschland, Osterreich und der Schweiz. Bd V1I, Part 2, Rabenhorst, Leipzig : 845 p.
Lange-Bertalot (H.) \& Ruppel (M.). 1980.-A revision of some taxo nomically most problematic groups in Achnanthes Bory. imponant from the ecological point of view. Arch. Hydrob. suppl. 60. Algological studies $26: 1-31$.

Leclerca (L.) \& Fabbri (R.). 1982. - Flore el végétation algales des bassins oligotrophes du bassin de la Schwalm (Betgique. Province de Liège). Bull. Soc. Bor. Belg.. 115; 53-68.

Le Cohu (R.). 1982.- Les especes endemiques de Diatomées aux íles Kerguelen. C.N.F.R.A., $51: 3542$.

Maillard (R.). 1978. - Contribution a la connaissance des Diatomées d'eau douce de la Nouvelle-Calédonie. Cah. O.R.S.T.O.M., sér. Hydrobiol. XII (2): 143-172.

Manguin (E.). 1962. - Contribution a la connaissance de la flore diatomique de la Nouvelle Calédonie. Mém. Mus. Hist. Nat. Paris, Nouv. sér., B, Bou., XII (1): 140.

Mc Intire (C.D.) \& Reimer (C.W.). 1974.- Some marine and brackish-water Achnanthes from Yaquina Estuary. Oregon (U.S.A.) Botanica Marina, XVII : 164-175.

Moss (M.O.) \& Carter (J.R.). 1982. $\rightarrow$ The resurrection of Achnanthes rusirata Ostrup. Bacillaria, $5: 157-164$.

Patrick (R.) \& Reimer (C.W.). 1966.- The Dialoms of the United States exclusive of Alaska and Hawai. Vol. 1. Acad. Nat. Sc. Philadephia, Monog. $13 ; 668$ p.

Pierre (J.F.) 1977. - Les algues des eaux courantes de l'île de la Possession (Archipel Crozet). Bull. Soc. Phycol. France, 22 : $79-86$

Schoeman (F.R.) \& Ashton (P.J.). 1982. - The diatom flora in the vicinity of the Pretoria Salt Pan. Transvaal. Republic of South Africa I. Nova Hedwigia, $73: 21-54$

Schoman (F.R.) \& Ashton (P.J.). 1983.- The diatom flora in the vicinity of the Pretoria Salt Pan. Transvaal, Republic of South Africa. Part II. S. Afr. Tydskr. Plantk., $2: 191-201$.

Schrader (H.J.). 1974.- Types of raphe structure in the dia coms. Nova Hedwigia 45 : $195-230$

Simonsen (R.). 1979.- The diatom system : ideas on phylogeny. Bacillaria, $2: 9.71$.

Sovereign (H.E.). 1958. - The diatoms of Crater Lake-Oregon Trans. American Micr. Soc. 77 (2): 96-134, 4 pl.

Therezien (Y.). 1976.- Algues d'eau douces des îles Kerguelen et de l'archipel Crozet (à l'exclusion des Diatomées). C.N.F.R.A. $41: 21-59$.

Therezein (Y.) \& Coute (A.). 1977.- Algues d'eau douce des iles Kerguelen et Crozet (à J'exclusion des Diatomes). C.N.F.R.A., 43 : $1.91,19 \mathrm{pl}$.

Tyler (P.A.). 1972,- Reconnaissance limnology of subantarctic islands. I. Chemistry of lake waters from Macquarie islands and the Iles Kerguelen. Int. Rev. Ges. Hydrobiol. Hydrog., 57 (5) : 759.778 